\title{
Benchmark solutions for natural convection flows in vertical channels submitted to different open boundary conditions ${ }^{\text {th }}$
}

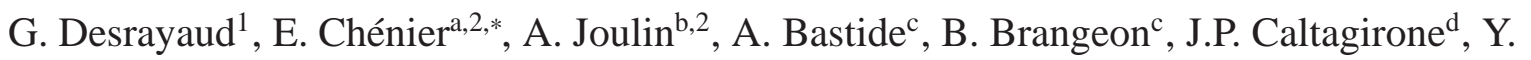 \\ Cherif $^{\mathrm{b}}$, R. Eymard ${ }^{\mathrm{e}}$, C. Garnier ${ }^{\mathrm{f}, \mathrm{g}}$, S. Giroux-Julien ${ }^{\mathrm{h}}$, Y. Harnane $^{\mathrm{i}}$, P. Joubert ${ }^{\mathrm{j}}$, N. Laaroussi ${ }^{\mathrm{k}}, \mathrm{S}$.

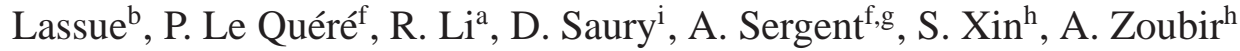 \\ ${ }^{a}$ Université Paris-Est, Laboratoire Modélisation et Simulation Multi Echelle, UMR-CNRS 8208, 5 Boulevard \\ Descartes, 77454 Marne-la-Vallée Cedex 2, France \\ ${ }^{b}$ Université Lille Nord de France, Laboratoire Génie Civil et géo-Environnement, EA 4515, Technoparc Futura, \\ 62400 Béthune, France \\ c PIMENT, 117 Avenue du Général Ailleret 97430 Le Tampon, France. \\ ${ }^{d}$ I2M - TREFLE Site ENSCBP - 16 Avenue Pey-Berland 33607 Pessac Cedex, France \\ eUniversité Paris-Est, Laboratoire d'Analyse et de Mathématiques Appliquées, UMR-CNRS 8050, 5 Boulevard \\ Descartes, 77454 Marne-la-Vallée Cedex 2, France \\ ${ }^{f}$ CNRS, LIMSI, BP 133, 91403 Orsay, France \\ ${ }^{g}$ UPMC, Univ Paris 06, F-75005, Paris, France \\ ${ }^{h}$ CETHIL UMR 5008 Bâtiment Sadi Carnot 9 Rue de la Physique INSA de LYON 69621 Villeurbanne Cedex, France \\ ${ }^{i}$ Institut PPRIME UPR CNRS 3346 - Université de Poitiers, ENSMA -Téléport 21 avenue Clément ADER, BP \\ 40109, 86961 Chasseneuil Futuroscope Cedex, France \\ ${ }^{j}$ LaSIE, Avenue Michel Crépeau 17042 La Rochelle Cedex 1, France \\ ${ }^{k}$ Ecole Supérieure de Technologie de Salé, Université Mohammed V-Agdal, Laboratoire d'Energétique, Matériaux et \\ Environnement (LEME), Avenue Prince Héritier, BP :227 Salé Medina, Maroc
}

\begin{abstract}
Comparison exercises have been carried out by different research teams to study the sensitivity of the natural convection occurring in a vertical asymmetrically heated channel to four sets of open boundary conditions. The dimensionless parameters have been chosen so that a return flow exists at the outlet. On the whole, results provided by the partners are in good agreement; benchmark solutions are then defined for each of the boundary conditions. Whilst the local and average Nusselt numbers based on the entrance temperature do not depend much on conditions applied in the aperture sections, the net fluid flow rates crossing the channel and the characteristics of the recirculation cells are highly influenced. But we proved that these modifications of flow patterns do not alter significantly the fluid flow rates leaving the channel through the exit section.
\end{abstract}

Keywords: Natural convection, Vertical channel, Recirculation flow, Open boundary conditions, Benchmark solutions 


\section{Nomenclature}

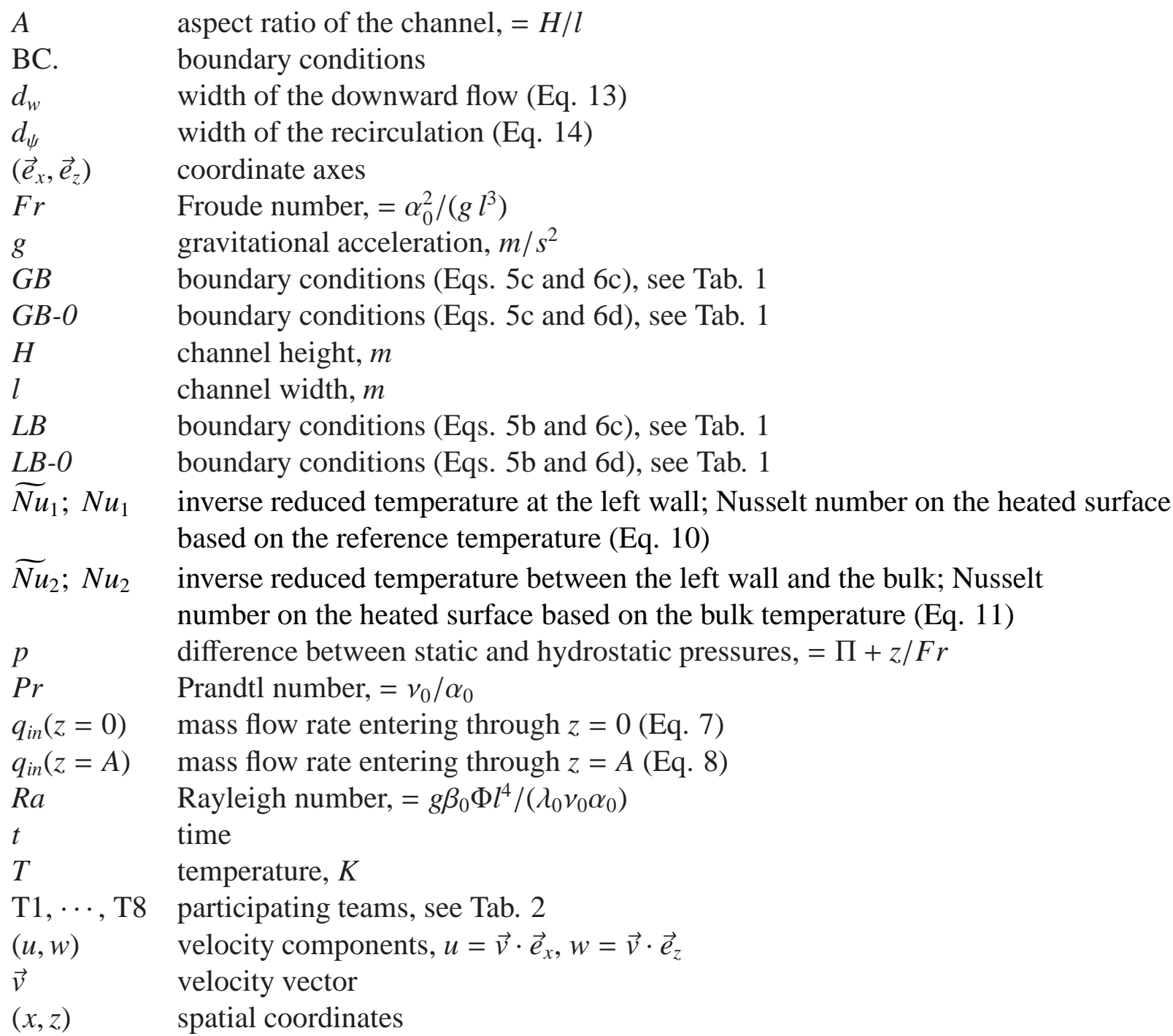

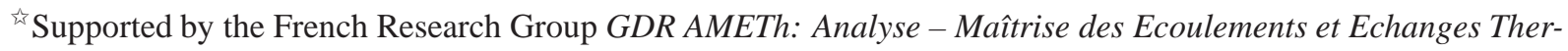
miques

${ }^{*}$ Corresponding author

Email addresses: Eric.Chenier@univ-paris-est.fr(E. Chénier), Annabelle.Joulin@univ-artois.fr (A. Joulin)

${ }^{1}$ To the memory of Gilles Desrayaud, deceased in January 2009

${ }^{2}$ Coordinators of the numerical exercise
} 

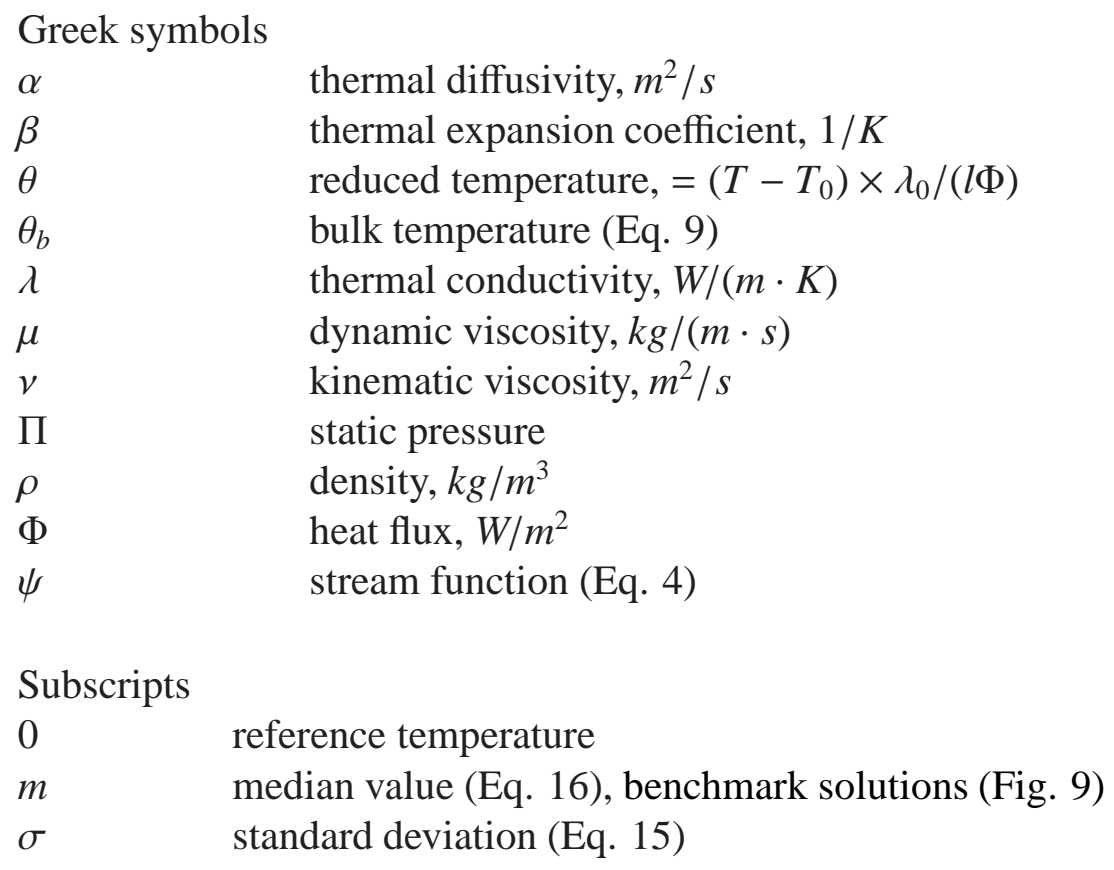

Others

\langle\rangle$\quad$ ensemble average (Eq. 15)

\section{Introduction}

Heat transfer and fluid flows driven by natural convection in open channels have been extensively studied over the last past decades, for vertical or inclined configurations. This great interest raised by this subject stems from its wide range of practical applications such as solar chimney, solar energy collectors, Trombe walls, or the cooling of electronic components and many others [1-4].

Since the precursory experimental works performed by Elenbaas in 1942 [5], who determined the different flow regimes versus a modified Rayleigh number or Elenbaas number (Rayleigh number calculated on the channel-chimney width divided by the aspect ratio), natural and mixed convections have been widely studied in open channels, both numerically and experimentally. Because the aim of this paper is to focus our attention on the influence of the boundary conditions applied in the apertures of the channel, and then to define benchmark solutions, the detailed description of the numerous contributions dealing with natural or mixed convection in vertical channels is transferred to few relevant and recent papers chosen to provide complete reviews of this topic (see [1, 6-13] and references therein). Among the first numerical simulations, we can mention the contribution of Bodoia and Osterle [14] who investigated fluid flows and heat transfer occurring through isothermal vertical plates. Their results were in good agreement with the Elenbaas experimental data [5]. Since then, and despite the plenty of numerical studies, the choice of 
the boundary conditions for open cavities is still a delicate issue.

For thermal natural convection, the distribution of the total heat fluxes conveyed by the fluid flow through the open boundaries depends on heat transfered at walls but also on physical conditions prevailing in the surroundings, on both sides of the apertures. It results a close coupling between the dynamic and thermodynamic variables inside and outside the channel. Thus, the thermal and kinematic inlet/outlet boundary conditions cannot be a priori prescribed without accounting for the surrounding conditions $[6,15,16]$. Finally, the thermally driven channel behaves as a thermal engine by converting the thermal gradient into a fluid flow. This corresponds to the socalled thermosyphon effect. Although thermal natural convection was only considered here-above, this applies for general mass transfer, whatever the origin of the density variation may be.

To overcome the issue of choosing the boundary conditions in inlet/outlet sections, some authors proposed to extend the computational domain, upstream and/or downstream the channel. Thus, the boundary conditions are pushed away from the true inlet/outlet sections of the channel. One of the ideas leading to the displacement of the open boundaries far from the channel limits is to reduce, as much as possible, the effect of an unawareness of the "real" boundary conditions. St. Venant's principle, often invoked in solid mechanics, may also be used in fluid mechanics: if the artificial boundaries are placed sufficiently far away from the channel apertures, the velocity and temperature distributions at the entrance/exit of the channel are no longer affected by the applied boundary conditions [17]. The issue now raised is how far the artificial open extensions must be placed in order that the physical quantities in the inlet/outlet sections become insensitive to the conditions set at boundaries. Although this approach seems attractive, the increase in size of the computational domain proves to be expensive, both in memory and in computational time. For these reasons, the domain extensions are often either relatively reduced or large but coarsely discretized. The shapes of the walls at the entrance region, with sharp angles or smooth rounded surfaces, affect also significantly the fluid flows and heat transfer. Using entrance walls with right angles, Naylor et al. [18] predicted a fluid separation at the channel inlet which is approximatively correlated with the dimensional flow rate. Their inlet boundary conditions were based on JeffreyHamel flow which consists in a similarity solution of isothermal flow caused by the presence of a source or sink at the point of intersection of two walls. Kettleborough [17], with a parabolic approximation, and Nakamura et al. [19], with a full elliptic model, used boundary conditions which physically correspond to fully developed flow entering a channel with a large sudden section reduction. They also found a separation of the boundary layer, but at some distance from the leading edge at the entry to the channel. This issue was reconsidered in a recent paper by Boetcher and Sparrow [20] who studied buoyancy-induced flow in an horizontal open-ended cavity. They examined the impact of the size of the extended domain, the boundary conditions on its surfaces, and the mesh density required to achieve high accuracy. Just as the upstream extensions may modify the flow and heat transfer in the channel-chimney, the increase in the computational downstream domain may lead to modifications in the draft height and thermal transfer, for instance by adding adiabatic extensions in order to enhance the sucking up of the chimney [21].

Another way to sidestep the question of choosing open boundary conditions in the entrance/exit sections of the channel is to encapsulate it into a virtual enclosure [22, 23]. As a result, the boundaries are solid walls on which it is easy to prescribe dynamical conditions. However, thermal boundary conditions are not so easy to define since they may disturb the thermal field around the 
channel and produce spurious natural recirculation cells.

The different strategies described here-above rest on an increase in size of the computational domain, and therefore, in computational memory storage and CPU time. An alternative is to restrict simulations to the channel height only, and to model the thermal and dynamical boundary conditions at the exact inlet/outlet sections. In comparison with numerical results by Bodoia and Osterle [14], Aihara [15] proposed to link the pressure to the mass flow rate. This formulation permits to substitute the unknown inlet velocity by a relation based on pressures. This writing is related to a simplified model of the upstream fluid flow sucked by the pressure drop produced in the channel aperture. In the upstream region, the fluid motion is then assumed incompressible, isothermal, inviscid and stationary. Dalbert et al. [16] were among the first to deal with this pressure drop which corresponds physically to the energy per volume unit required to bring the fluid from rest far upstream to the entrance section. Using Jeffrey-Hamel flow extension at the entrance, Naylor et al. [18] confirmed the relationship between the kinetic energy and the pressure drop in the inlet section of the channel.

Since a long time, simulations performed by numericists rely on reference solutions to check both the validity of discretization schemes and their numerical implementations. For laminar flows and closed systems, such benchmark solutions are well documented. For example, one may mention the lid driven cavity for isothermal fluid flows and the Rayleigh-Bénard problem or the differentially heated cavity (window problem) for flows driven by buoyancy. But to our best knowledge, and despite very numerous numerical references, such a work is still lacking for free convection flowing through open boundaries. This issue was already addressed by the French community dealing with thermal sciences for a geometrical model restricted to the channel height [24]. But this first attempt to define reference solutions turned out to be unsuccessful: the gaps between results provided by the research teams were too large, and no reference solution was defined. A possible explanation of these discrepancies was recently proposed in [25]. For one particular set of boundary conditions, Le Quéré showed that the use of Neumann type boundary conditions may give one or several nontrivial combinations of velocity-pressure fields which satisfy the homogeneous Stokes operator, in addition to the unavoidable constant pressure mode. This recognition leads him to propose an algorithm in which the solution is sought as a combination of particular solution of the inhomogeneous Stokes or unsteady Stokes problem, plus a linear combination of the modes of the Stokes kernel so as to satisfy the pressure drop between the inlet and outlet.

Considering the previous works $[24,25]$ and the obvious interest of the numericists community to have reference solutions for free convection with open boundaries, we reformulated the test case problem [24] on the basis of four boundary conditions frequently met in such studies. This new approach leads us to study the sensitivity of fluid flows and heat transfer to the different prescribed boundary conditions.

The purpose of this paper is then twofold. First, it aims at contributing to the numerical study of natural convection in vertical channel by considering some sets of boundary conditions, but always restricting the geometry to the physical domain, without any downstream or upstream domain extensions. These boundary conditions are, for example, implemented in some commercial codes or free packages solving the Navier-Stokes and energy equations. The second objective concerns 
the definition of reference solutions that could be very useful in the community of numericists for validating the numerical schemes in open systems, when flows are driven by natural or even mixed convection. In order to propose a numerical exercise, both relevant and numerically delicat, and to emphasize the effect of boundary conditions on fluid flow and heat transfer, we focused our attention on a problem which gives rise to a recirculation flow at the exit of the channel [26, 27].

The rest of the paper is organized into two main sections followed by a conclusion. Section 2 provides, first the mathematical model with the four sets of boundary conditions, and then describes the different variables used for comparisons. Section 3, which deals with the results and their discussions, is subdivided into three subsections. A qualitative description of the flow and temperature fields is first given. Then, the results of the different research teams are synthesized and commented; reference solutions are defined. Finally, the influence of the different boundary conditions on heat and fluid flow is presented and analyzed. A conclusion summing up the key points of this contribution ends the paper.

\section{Description of the test cases}

\subsection{Heat and fluid flow equations}

A vertical parallel plate channel of width $l$ and height $H$ is formed by two walls, one partially heated at a constant and uniform heat flux $\phi$ on its half middle section and the remaining walls are adiabatic (see fig. 1) [26]. The fluid flow is assumed laminar and two-dimensional. The thermal radiations and the heat conduction inside the solid walls are disregarded. Accounting for the small relative temperature difference occurring between the heated wall and the aperture, the NavierStokes and energy equations are expressed with the Boussinesq approximation. The problem to be numerically solved is restricted to the channel height. The governing flow and heat transfer equations, written in dimensionless form, read:

$$
\begin{gathered}
\vec{\nabla} \cdot \vec{v}=0 \\
\frac{\partial \vec{v}}{\partial t}+\vec{\nabla} \cdot(\vec{v} \otimes \vec{v})=-\vec{\nabla} p+\operatorname{Pr} \nabla^{2} \vec{v}+\operatorname{Ra} \operatorname{Pr} \theta \overrightarrow{e_{z}} \\
\frac{\partial \theta}{\partial t}+\vec{\nabla} \cdot(\vec{v} \theta)=\nabla^{2} \theta
\end{gathered}
$$

with $\vec{v}=u \overrightarrow{e_{x}}+w \overrightarrow{e_{z}}$ the dimensionless velocity vector, $p=\Pi+z / F r$ the dimensionless departure of the static pressure from the hydrostatic pressure, and $\theta=\lambda_{0}\left(T-T_{0}\right) /(\phi l)$ the reduced dimensionless temperature. The reference temperature $T_{0}$ is set to the temperature of the surroundings. Thermal conductivity, thermal and viscous diffusivity and thermal expansion coefficient are expressed at $T_{0}$ by $\lambda_{0}, \alpha_{0}, v_{0}$ and $\beta_{0}$ respectively. The length, velocity and pressure are scaled by $l, \alpha_{0} / l$ and $\rho_{0} \alpha_{0}^{2} / l^{2}$. The dimensionless parameters governing the fluid flow and heat transfer are the Prandtl number $\operatorname{Pr}=v_{0} / \alpha_{0}$, the Rayleigh number $R a=g \beta_{0} \phi l^{4} /\left(\lambda_{0} v_{0} \alpha_{0}\right)$ and the aspect ratio of the channel $A=H / l$. The dimensionless streamfunction is defined as usual by

$$
\frac{\partial \psi}{\partial z}=u, \quad \frac{\partial \psi}{\partial x}=-w
$$




\subsection{Boundary conditions}

Four sets of boundary conditions are referenced in this paper. In each case, the conditions at the solid walls are the same; only the boundary conditions at the inlet and outlet sections are modified.

\section{Boundary conditions at solid plates.}

- Left wall, $x=0$,

$$
\begin{cases}\forall z \in] 0 ; A[, & \vec{v}(0, z)=\overrightarrow{0} \\ \forall z \in] 0 ; A / 4[\cup] 3 A / 4 ; A[, & \frac{\partial \theta}{\partial x}(0, z)=0 \\ \forall z \in] A / 4 ; 3 A / 4[, & \frac{\partial \theta}{\partial x}(0, z)=-1\end{cases}
$$

- Right wall, $x=1, \forall z \in] 0 ; A[$,

$$
\left\{\begin{array}{l}
\vec{v}(1, z)=\overrightarrow{0} \\
\frac{\partial \theta}{\partial x}(1, z)=0
\end{array}\right.
$$

Channel inlet, $z=0, \forall x \in] 0 ; 1[$,

$$
\left\{\begin{array}{l}
u(x, 0)=0 \\
\frac{\partial w}{\partial z}(x, 0)=0 \\
\theta(x, 0)=0
\end{array}\right.
$$

Two pressure boundary conditions are studied. They result from Bernoulli's theorems which assume stationary, incompressible and inviscid fluid flows.

- Local Bernoulli relation $(L B)$ :

$$
\forall x \in] 0 ; 1\left[, \quad p(x, 0)=-\frac{1}{2}(w(x, 0))^{2}\right.
$$

- Global Bernoulli relation $(G B)$ :

$$
\forall x \in] 0 ; 1\left[, \quad p(x, 0)=-\frac{1}{2}\left(q_{\text {in }}(z=0)\right)^{2}\right.
$$

where $q_{\text {in }}(z=0)=\int_{0}^{1} w(x, 0) d x$ denotes the dimensionless mean velocity or flow rate.

Channel outlet, $z=A$. Although the designation "outlet" (or "exit") is somewhat incorrect in our problem, since fluid partially enters through the top boundary and creates a recirculation flow, but for sake of simplicity, the use of this term is however kept. To establish the boundary relations, it is then necessary to distinguish the conditions corresponding to entering $(w(x, A)<0)$ and exiting $(w(x, A)>0)$ fluid flows. Furthermore, two pressure conditions are studied when the fluid flows out of the channel. 
- Exiting fluid:

$$
\text { For all } x \in] 0 ; 1\left[\text {, such that } w(x, A) \geq 0, \quad\left\{\begin{array}{l}
u(x, A)=0 \\
\frac{\partial w}{\partial z}(x, A)=0 \\
\frac{\partial \theta}{\partial z}(x, A)=0 \\
p(x, A)=0
\end{array}\right.\right.
$$

- Entering fluid:

$$
\text { For all } x \in] 0 ; 1\left[\text {, such that } w(x, A)<0, \quad\left\{\begin{array}{l}
u(x, A)=0 \\
\frac{\partial w}{\partial z}(x, A)=0 \\
\theta(x, A)=0
\end{array}\right.\right.
$$

with one of the two following pressure boundary conditions:

- Local relation

For all $x \in] 0 ; 1\left[\right.$, such that $w(x, A)<0, \quad p(x, A)=-\frac{1}{2}(w(x, A))^{2}$

- Uniform Pressure

$$
\text { For all } x \in] 0 ; 1[\text {, such that } w(x, A)<0, \quad p(x, A)=0
$$

The four sets of boundary conditions are synthesized with notations ( $L B, L B-O, G B$ and $G B-O)$ according to the rule given in Tab. 1. From a mnemonic point of view, the notations $L B$ (Local Bernoulli, Eq. (5b)) and $G B$ (Global Bernoulli, Eq. (5c)) designate the boundary conditions at the lower channel aperture. The addition of the suffix -0 indicates that the outlet pressure is uniformly set to zero (see Eqs. (6a) and (6d)), otherwise local expressions are applied for the return flow (Eqs. (6a) and (6c)). It must be emphasized that the pressure boundary condition is a continuous function of the abscissa, even if a reversal flow occurs at the channel exit.

Before introducing the variables which will lead to quantitative comparisons, let us perform some remarks about thermal radiation. First, and as announced at the beginning of Sec. 2.1, the thermal radiation has been neglected, since the aim of the paper is to focus the analysis on pressure boundary conditions and the definition of benchmark solutions in the scope of pure natural convection. However, from a physical point of view, working with transparent fluids like air gas makes the surface temperature sensitive to the surface radiation phenomena. In that case, it is worth pointing out that, despite small relative temperature differences, surface radiations may strongly modify the flow characteristics, even if the emissivities are very small [28]. Consequently, the radiation exchanges must always be taken into account in the numerical model if comparisons with experimental data are planned (see the works by Li et al. [29]). 


\subsection{Monitored variables}

Comparisons have been carried out for mean and local quantities at different discrete vertical coordinates located in the heated region and in the downstream adiabatic domain, namely for $z \in\{3 A / 8 ; A / 2 ; 5 A / 8 ; 3 A / 4 ; 7 A / 8 ; A\}$. These concern some

\section{dynamic aspects:}

- the mass flow rate entering the channel through the bottom section $z=0$

$$
q_{\text {in }}(z=0)=\int_{0}^{1} w(x, 0) d x
$$

- the mass flow rate entering into the channel through the top section $z=A$

$$
q_{\text {in }}(z=A)=\int_{0}^{1} \frac{|w(x, A)|-w(x, A)}{2} d x
$$

\section{thermal variables:}

- the bulk temperature

$$
\theta_{b}(z)=\frac{1}{q_{\text {in }}(z=0)} \int_{0}^{1} w(x, z) \theta(x, z) d x
$$

- the inverse of the temperature at the left wall

$$
\widetilde{N u}_{1}(z)=\frac{1}{\theta(0, z)}
$$

whose expression corresponds to the local Nusselt number $N u_{1}(z)$ on the heated wall only, with a reference temperature equal to the surrounding value; $N u_{1}(z>3 A / 4)=0$.

- the inverse of the difference between the temperature at the left wall and the bulk temperature

$$
\widetilde{N u}_{2}(z)=\frac{1}{\theta(0, z)-\theta_{b}(z)}
$$

whose expression represents the local Nusselt number $\mathrm{Nu}_{2}(z)$ on the heated wall only, with the bulk temperature as reference temperature; $N u_{2}(z>3 A / 4)=0$.

- the average Nusselt numbers on the heated wall

$$
\overline{N u_{i}}=\frac{2}{A} \int_{A / 4}^{3 A / 4} N u_{i}(0, z) d z, \quad i \in\{1,2\}
$$

\section{characterization of flow patterns:}


- the length of the downward flow in $z$-section. For $z$ given, if there exists $0<x_{w}(z)<1$ such that $w\left(x_{w}(z), z\right)=0$, then

$$
d_{w}(z)=1-x_{w}(z)
$$

- the recirculation length in $z$-section. For $z$ given, if there exists $0<x_{\psi}(z)<1$ such that $\psi\left(x_{\psi}(z), z\right)=\psi(1, z)$, then

$$
d_{\psi}(z)=1-x_{\psi}(z)
$$

with the streamfunction defined in Eq. (4).

\section{Results and discussions}

The numerical exercise has been carried out for $R a=5 \times 10^{5}, A=10$ and air as working fluid with $\operatorname{Pr}=0.71$.

Results were obtained by eight partners which are parts of different laboratories: CETHIL, I2M-TREFLE, LAMA, LaSIE, LEME, LGCgE, LIMSI, MSME, PIMENT and PPRIME. Five of the numerical codes were developed by the research teams, two are free CFD software packages (Aquilon $\AA$, FDS) and the last one is commercial (Fluent $($ )). Table 2 indicates, for the different partners, the name of the laboratories involved, and some characteristics of the numerical schemes. More details about the numerical method are provided in the appendix section.

\subsection{Qualitative description of the fluid flow and temperature field}

The fluid flow and the temperature field, illustrated in Fig. 2 for boundary conditions $L B$ (Tab. 1), are qualitatively almost the same whatever the boundary conditions considered in this paper. The fluid is heated along the mid-height of the central section of the left wall by a uniform and constant heat flux $\Phi$ (Fig. 2(d)). Consequently, the fluid rises up due to the density variations and creates a dynamic boundary layer along the left plate (Fig. 2(c)). Due to too large head loss in the upstream adiabatic part, the feeding of this dynamic boundary layer cannot exclusively happen by an air supply coming from the lower aperture of the channel and then an incoming of fluid through the top open section of the channel is created over a width $d_{w}(z=A)$ (Fig. 2(c)). Therefore, a recirculation flow occurs with a measured size at the channel exit $d_{\psi}(z=A)$ (Fig. 2(a)).

It must be underlined that the field of the horizontal component (Fig. 2(b)) displays positive and negative values in the vicinity of the left and right corners of the entrance region. These patterns are typically met with BC. (5b), i.e. when the inlet pressure is set to the local dynamic pressure. If the pressure boundary condition in the aperture is kept uniform BC. (5c), these local variations in the horizontal velocity do not exist. The small fluid pocket, defined by $u(x, z)>0$ and located near the center of the channel exit section, has been identified by all the participants working on $L B$ problem. When other boundary conditions are applied, it disappears. 


\subsection{Comparison of numerical contributions}

Figures 3, 4, 5 and 6 provide $\widetilde{N u}_{1}(z)$ (Eq. 10) and $\widetilde{N u}_{2}(z)$ (Eq. 11), viz the local Nusselt numbers $N u_{1}(z)$ and $N u_{2}(z)$ when $A / 4 \leq z \leq 3 A / 4$, the local sizes of both the return flow $d_{w}(z)$ (Eq. 13) and the recirculation flow $d_{\psi}(z)$ (Eq. 14), the local bulk temperature $\theta_{b}(z)$ (Eq. 9), and four overall values defined by the mean Nusselt numbers on the heated plate $\overline{N u}_{1}$ and $\overline{N u}_{2}$ (Eq. 12), and the flow rate penetrating the channel through the bottom $q_{i n}(z=0)$ (Eq. 7) and the top $q_{i n}(z=A)$ (Eq. 8) sections, for boundary conditions $G B-0, L B-O, G B$ and $L B$. The results of each research team have been collected and graphically represented using histograms with the corresponding numerical values accurate within 4 significant digits, and topped by three statistical quantities, the average $(\langle x\rangle)$, the standard deviation $\left(x_{\sigma}\right)$ and median value $\left(x_{m}\right)$ defined as follows:

$$
\begin{gathered}
\langle x\rangle=\frac{1}{n} \sum_{i=1}^{n} x_{i}, \quad x_{\sigma}=\sqrt{\left\langle x^{2}\right\rangle-\langle x\rangle^{2}} \\
x_{m}= \begin{cases}x_{(n+1) / 2}, & \text { if } n \text { is odd, with } x_{1} \leq \cdots \leq x_{n} \\
\left(x_{n / 2}+x_{n / 2+1}\right) / 2, & \text { if } n \text { is even, with } x_{1} \leq \cdots \leq x_{n}\end{cases}
\end{gathered}
$$

with $x$ any of the recorded variables mentioned here-above. When results are in excellent agreement, the mean and median values are very close, and the standard deviation is small in comparison with the average value. In that case, the mean or median values provide very good approximations of the reference solution. In contrast, if some results depart significantly from the other contributions, the mean value can differ substantially from the median value. This bias which is introduced is related to the relative small number of partners involved in the comparison exercice. In that case, the median value is proved to be better representative of the results than the average value. Therefore, the reference results are defined by the measure of the median $x_{m}$.

On the whole, the results provided by the different teams are in quite good agreement. A deeper insight is now proposed for the 4 sets of boundary conditions.

We first consider boundary conditions $G B-O$ and the associated results presented in Fig. 3 . We notice that the standard deviations are small $\left(x_{\sigma} /\langle x\rangle<0.6 \%\right)$ for the average Nusselt numbers $x \equiv \overline{N u}_{1}$ and $\overline{N u}_{2}$ (Fig. 3(f)). This good agreement between the different teams is confirmed when we look at the local values $\widetilde{N u}_{1}(z)$ (Fig. 3(a)) and $\widetilde{N u}_{2}(z \leq 3 A / 4)$ (Fig. 3(b)), since the average discrepancy does not exceed $1.7 \%$. Beyond $z=3 A / 4$, the agreement between the participants becomes all the more worse since one goes closer to the top section $\left(\widetilde{N u_{2 \sigma}} /\left\langle\widetilde{N u_{2}}\right\rangle \approx 7 \%\right.$ for $z=A$.). Let us recall that $\widetilde{N u_{1}}(z)$ is inversely proportional to $\theta(0, z)$ (Eq. 10), and therefore its dispersion results necessary from disagreements in the temperature distribution along the left plate. The second definition $\widetilde{N u}_{2}(z)$ is inversely proportional to the difference between the wall temperature and the bulk temperature $\theta_{b}(z)$ (Eq. 11). Thus, the more the temperature gap decreases, the more this value will be sensitive to the computational uncertainties. The variations of the bulk temperature $\theta_{b}(z)$ (Fig. 3(e)) are in good accordance, except for two families of results that were provided by teams T1 and T6. This discrepancy shown on the bulk temperature is found on the dynamical variables as well. Indeed, their two flow rates $q_{i n}(z=0)$ and $q_{i n}(z=A)$ (Fig. 3(f)), their sizes of the recirculation flow $d_{\psi}(z)$ (Fig. 3(d)) and their lengths corresponding to the fluid 
flow penetrating the channel through the top section $d_{w}(z)$ (Fig. 3(c)) differ slightly from the data provided by the other participants: whereas both the width of the recirculation $d_{\psi}(z)$ looks smaller and the return flow seems to penetrate less deeply into the channel for team $\mathrm{T} 6$, the perfect opposite behavior is observed for results given by T1. Attempts of explanation for these departures are now given.

The numerical method developed by T6 results from a recent analysis of the discrete Stokes problem based on a staggered grid formulation with a projection method to uncouple the velocity and pressure $[25,30,31]$. The usual homogeneous Neumann boundary conditions applied to the pressure increment may lead to an increase of the kernel size of the Stokes operator making the solution of the full nonlinear equations indefinite. This arises in particular when Neumann type boundary conditions are imposed on the velocity component normal to the inlet and outlet boundaries. The natural convection problem under consideration falls into this category. An appropriate algorithm has been proposed that is derived from the principle of superposition. The final numerical solution is a linear combination of a particular solution provided by a conventional finite volume scheme and of the (velocity, pressure) modes belonging to the Stokes kernel. These novel works clearly raise the issue of the choice of the artificial boundaries conditions but also of their numerical implementation. As it has been shown in $[25,30,31]$ the numerical method used by T6 leads to control two parameters in the linear combination of the Stokes's kernel modes. To determine them, two specific constraints of the flow have to be specified. The choice has been made to impose the mean pressure in the outlet section equal to zero and the mean pressure in the inlet section equal to $-\left(q_{\text {in }}(z=0)\right)^{2} / 2$. The discrepancies observed on $G B-0$ results are then due to the fact that T6 imposed mean pressures on the inlet and outlet sections and not uniform pressures as requested by Eqs. 5c and $6 \mathrm{~d}$. Thus, T6 solved a neighboring physical problem which seems less restrictive for the flow field than $G B-O$ does. Consequently, the comparison with the results by $\mathrm{T} 6$ shows the great dependence of the problem on the pressure boundary conditions.

The explanations for the departures of $\mathrm{T} 1$ results are unclear, but some specific points can be pointed out. First, the FDS software used by T1 rests on a low Mach number approximation, suitable for low speed thermally-driven flows with large temperature variations. However, this model should provide results in accordance with those obtained with Boussinesq approximation since the maximal relative temperature never exceeds $\approx 5 / 290=1.7 \%$. Another remark can be drawn about the accuracy of computations. Indeed, the mass flow rate shows a slight increase of about $0.1 \%$ from the inlet to the outlet sections. Although this variation may be considered as negligible, the natural convection flows in open channel are proved to be very sensitive to numerical errors, and specially to mass conservation. Thus, this lack of numerical accuracy may explain the small disagreements noticed on these results.

For boundary conditions $L B-O$ (Fig. 4), the data given by teams T2, T3 and T8 are overall in good agreement: $x_{\sigma} /\langle x\rangle$ is less or equal than $1 \%$ for $x \equiv \widetilde{N u_{1}}(z), \widetilde{N u_{2}}(z \leq 5 A / 8), d_{w}(z \geq 3 A / 4)$, $d_{\psi}(z \geq 3 A / 4), \theta_{b}(z)$ and also for the mean Nusselt numbers and mass flow rates (Fig. 4(a)-(f)). Lastly, the ratio between the standard deviation and the average value is about $2 \%$ for $d_{w}(5 A / 8)$ and $d_{\psi}(5 A / 8)$ and reaches up to $22 \%$ for $\widetilde{N u}_{2}(A)$ ! This latter very large gap is due to both the bulk temperature accuracy $(\approx 1 \%)$ and the small temperature difference between the wall and bulk. To support this assertion, let us evaluate roughly $\widetilde{N u}_{2}(z=A)$ from the bulk and wall 
temperatures at $z=A$. The wall temperature at the channel exit is derived from the definition of $\widetilde{N u_{1}}$ (Eq. 10), the mean value $\left\langle\widetilde{N u}_{1}(A)\right\rangle=11.69$ and the relative standard deviation $\widetilde{N u}_{1 \sigma}(A) /\left\langle\widetilde{N u}_{1}(A)\right\rangle=0.1 \%: \theta(x=0, z=A)=1 / 11.69 \pm 0.1 \%$. One gets $\theta(x=0, z=A)-\theta_{b}(A)=$ $(0.08554 \pm 0.1 \%)-(0.08298 \pm 0.9 \%)=2.56 \times 10^{-3} \pm 8.3 \times 10^{-4}$, that is an error of $32 \%$. Finally, one has $\widetilde{N u}_{2}(A) \approx 390 \pm 32 \%$, namely an average value very close to that reported in Fig. 4(b) for $z=A$, but with an error about $45 \%$ larger than the corresponding standard deviation value $\left(\widetilde{N u}_{2 \sigma}(A) /\left\langle\widetilde{N u}_{2}(A)\right\rangle=22 \%\right)$.

The results provided by teams T2, T3 and T8 for $G B$ boundary conditions (Fig. 5) as well as those given by T2, T3, T7 and T8 for $L B$ boundary conditions (Fig. 6) are in excellent agreement, both for the thermal and the dynamical quantities. The ratios between the standard deviations and the mean values are less, or even much less than $1 \%$, except $d_{w_{\sigma}}(3 A / 4) /\left\langle d_{w}(3 A / 4)\right\rangle \approx 1.6 \%$ and $q_{i n_{\sigma}}(z=A) /\left\langle q_{i n}(z=a)\right\rangle \approx 1.9 \%$ for $G B$ (Fig. 5(c) and (f)) and $d_{\psi_{\sigma}}(5 A / 8) /\left\langle d_{\psi}(5 A / 8)\right\rangle \approx 1.2 \%$ for $L B$ (Fig. 6(d)).

\subsection{Effect of boundary conditions on heat transfer and fluid flow}

Figure 7 displays the vertical component of the velocity in different horizontal sections of the channel, for the pressure outlet condition Eq. (6c) and two pressure inlet conditions Eq. (5b) and Eq. $(5 c)$.

Major differences are shown in the entrance section. Indeed, when the inlet pressure is based on the local kinetic energy per volume unit (Fig. 7(a)), the velocity consists essentially of a flat profile far from the solid walls and it turns out to be of parabolic shape when the pressure is uniform in the entrance section (Fig. 7(b)). However, in both situations, the velocity profiles become almost parabolic at the entrance of the heated region at $z=A / 4$ (filled squares). The occurrence of an already established isothermal flow in the aperture section of the channel (see Fig. 7(b)) is counterintuitive and somewhat questionable. Although the issue of choosing boundary conditions capable to mimic the correct physical conditions is out of the scope of the present contribution, we can however notice that preliminary experimental measurements [32] performed on inlet velocity seem rather indicate uniform profiles in agreement with Fig. 7(a) obtained with BC. (5b). Substituting outlet BC. (6c) by BC. (6d) does not alter the inlet velocity profiles.

The development of the dynamic boundary layer along the left wall as well as the back flow entering through the top section at $z=A$ are clearly visible in Fig. 7 (open triangles). We can also notice that the return flow penetrates deeper into the channel for $L B$-boundary conditions: at $z=3 A / 4$ (filled circles), the magnitude of the downward fluid flow is slightly larger in Fig. 7(a) than in Fig. 7(b). This qualitative observation is confirmed by the accurate measurements of $d_{w}(z)$ and $d_{\psi}(z)$ which are reported in Fig. 9(c) and Fig. 9(d) and commented afterwards.

Figure 8 presents the pressure and vertical velocity profiles in the vertical median section between the parallel plates versus the vertical coordinate, for $L B, G B, L B-O$ and $G B-O$ problems. From Fig. 8(a), we see that the pressure is first decreasing in the lower adiabatic region because of the head loss occurring in the channel for isothermal fluid flows. Then, beyond $z=A / 4$, the buoyancy force becomes active and the slope of the pressure gradient gets reversed. Additional features are deduced from the spatial evolutions of these curves. We clearly see that the pressures $G B$ and $G B-0$ are linearly decreasing in the lower adiabatic region when BC. (5c) is applied in the aperture 
sections, what confirms the flow is dynamically established upstream the heated region (see also Fig. 7(b)). Above $z=7 A / 8$, the pressure curves show a "S"-shape behavior, all the more marked than the pressures in the inlet/outlet sections depend on the kinetic energy per volume unit. The occurrence of fully developed flows in the lower adiabatic regions for $G B$ and $G B-0$ cases are also confirmed by the constant vertical velocity profiles (Fig. 8(b)). The increases in $w(0.5, z)$, shown in Fig. 8(b) for $L B$ and $L B-O$ situations, are related to the dynamic boundary layer developments which slow down the flows close to the wall and accelerate the fluid motions at the center of the channels. But once the heated regions are reached, thermal boundary layers along the left surfaces grow and modify the kinetic boundary layers by increasing the fluid velocities due to buoyancy forces (see also Fig. 7). Since the flow rates must be conserved, it results some decreases in the vertical velocities at the centers of the channel.

Figure 9 gathers the whole statistical results for boundary conditions $L B-O, L B, G B-O$ and $G B$ together. Histograms and error bars stand for average and standard deviation values. Graphics are then topped by the mean values $\langle x\rangle$, plus the relative standard deviation error $\pm x_{\sigma} /\langle x\rangle$ reported in parentheses and expressed in permillage $(\% o)$. To emphasize the agreement or disagreement between participants, these values are colored in blue if $x_{\sigma} /\langle x\rangle<1 \%$ and in red otherwise. As announced in the first paragraph of Sec. 3.2, the reference solution is set to the median $x_{m}$ and its value, accurate within four significant digits, is reported below the corresponding histogram. Before considering the results in details, we can notice that $x_{\sigma} /\langle x\rangle$ are rather small, what indicates a good agreement between the results provided by the different partners.

Let us first analyze the thermal results. The comparison between the four studied cases $L B$ $0, L B, G B-0$ and $G B$, for the average Nusselt number $\overline{N u}_{1}$ (Fig. 9(f)) and $\widetilde{N u}_{1}(z)$ (Fig. 9(a)) shows a maximal relative gap of $3.2 \%$. However, when one considers the local Nusselt number along the heated surface using the bulk temperature as reference, the relative difference increases significantly with $z$ (Fig. 9(b)): $N u_{2}(z)[L B-O] / N u_{2}(z)[G B]-1$ is approximatively equal to $8 \%$ at $z=A / 2,14 \%$ at $z=5 A / 8,23 \%$ at $z=3 A / 4$. In the adiabatic region, this relative difference $\widetilde{N u}_{2}(z)[L B-0] / \widetilde{N u}_{2}(z)[G B]-1$ grows dramatically from $114 \%$ at $z=7 A / 8$ to more than $1000 \%$ at $z=A$. Nonetheless, the average Nusselt value on the heated section $\overline{N u}_{2}$ remains weakly affected by the boundary condition changes since the relative difference does not exceed $8 \%$. The local bulk temperature is also very sensitive to the adopted model (Fig. 9(e)), but, contrary to $\widetilde{N u}_{2}(z)$, the relative difference is almost constant with $z: \theta_{b}(z)[L B-O] / \theta_{b}(z)[G B]-1 \approx 43 \%$. This relative gap is easily explained by the following factors. Since the axial thermal diffusion is essentially negligible with respect to the transverse diffusion, it is easy to prove that the bulk temperature increases linearly in the heated section with a slope close to $1 / q_{i n}(z=0)$ and then it keeps constant in the adiabatic region. Therefore, the departure in the local bulk temperature is given by the relative difference obtained in the flow rate entering the channel at $z=0$, namely $q_{\text {in }}(z=0)[G B] / q_{\text {in }}(z=0)[L B-O]-1 \approx 43 \%$ (Fig. 9(f)).

In the second step, let us focus on the dynamical aspects. A first insight shows that the flow patterns generated by the natural convection as well as the flow rates are very sensitive to conditions imposed at the inlet/outlet of the channel sections (Figs. 9(c),(d) and (f)). The maximal relative gaps recorded at $z=A$ lie from about $18 \%$ for $d_{\psi}(z=A)$ and $d_{w}(z=A)$ up to $140 \%$ for $q_{i n}(z=A)$. A thoroughly study shows that applying uniform boundary conditions in the inlet section, namely 
BC. (5c), creates larger flow rates $q_{i n}(z=0)$ than configurations where boundary conditions are based on the local kinetic energy per volume unit (BC. 5b), i.e. $q_{i n}(z=0)[G B]>q_{\text {in }}(z=0)[L B]$ and $q_{\text {in }}(z=0)[G B-O]>q_{\text {in }}(z=0)[L B-O]$. Furthermore, if we compare in Fig. 8(a) the mean pressure drop in the entrance adiabatic region between $G B$ and $L B$, and between $G B-O$ and $L B-O$, we note that its value is larger if the inlet pressure is defined locally, what corresponds to the existence of a dynamic entry zone where the fluid flow evolves from a flat profile to a Poiseuille-like profile (Fig. 7(a)). We then deduce that the reduction in flow rate $q_{i n}(z=0)$ obtained with BC. (5b) versus BC. (5c) is due to head losses induced by the fluid flow development in the lower adiabatic region. Let us now consider the flow rates induced by the return flow for fixed inlet boundary conditions. Fig. 9(f) indicates that $q_{i n}(z=A)$ is reduced when the outlet pressure boundary conditions depend on the fluid flow direction (BC. 6c), i.e. $q_{i n}(z=A)[G B]<q_{i n}(z=A)[G B-0]$ and $q_{i n}(z=A)[L B]<q_{i n}(z=A)[L B-O]$. The decrease in this flow rate is also correlated with the shortening of the return flow $d_{w}$ (Fig. 9(c)) and of the recirculation cell $d_{\psi}$ (Fig. 9(d)). These behaviors are directly explained by the intensity of the pressure imposed to the return flow, since it is smaller with BC. (6c) than BC. (6d) and consequently less efficient to create an inflow circulation through the upper aperture. If we now compare the total flow rate leaving the channel, namely

$$
q_{\text {out }}=q_{\text {in }}(z=0)+q_{\text {in }}(z=A)
$$

we notice that $q_{\text {out }}[L B-0]<q_{\text {out }}[L B]<q_{\text {out }}[G B-0]<q_{\text {out }}[G B]$, what is in accordance with the behavior of $q_{\text {in }}(z=0)$ (Fig. 9(f)). While the maximal relative difference of the flow rate entering into the channel at $z=0$ and defined by

$$
\frac{q_{\text {in }}(z=0)[G B]}{q_{\text {in }}(z=0)[L B-0]}-1
$$

is about $43 \%$, the value of expression (18) falls down to $10 \%$ if $q_{\text {in }}(z=0)$ is substituted by $q_{\text {out }}$ (Eq. 17) instead. Therefore, the flow rate leaving the channel through the top section is almost the same whatever the boundary conditions are. This result is explained by the kind of flow crossing the channel apertures. Indeed, this flow rate aims to feed the dynamic boundary layer created by the wall heating and driven by the buoyancy force. And to do this, the fluid path fits to minimize the total head loss as follows:

- the inflow rate $q_{i n}(z=0)$ (resp. $q_{i n}(z=A)$ ) is maximal (resp. minimal) through the lower (resp. upper) aperture when both

- the velocity is fully developed all along the upstream adiabatic region (Eq. 5c), i.e. the upstream head loss is minimal,

- the pressure at the outlet acts against the appearance of a return flow (Eq. 6c) by producing a suction (negative pressure).

- the inflow rate $q_{\text {in }}(z=0)$ (resp. $q_{\text {in }}(z=A)$ ) is minimal (resp. maximal) through the lower (resp. upper) aperture when both

- an entry length exists in the upstream adiabatic region (Eq. 5b), i.e. the upstream head loss is maximal, 
- the pressure at the outlet promotes the growth of a return flow (Eq. 6d) by pushing the fluid into the channel (positive pressure).

This flow rate analysis confirms that the modification in the open boundary conditions acts significantly on the dynamic variables and less on the heat transfer on the heated section of the left plate. On the other hand, the Nusselt number $N u_{2}(z)$ clearly depends on the boundary conditions in the downstream adiabatic region. This sensitivity is due to its dependency into the bulk temperature $\theta_{b}(z)$ (or $q_{\text {in }}(z=0)$ ), which, in turn, is highly linked both to the flow structure and its intensity.

\section{Conclusion}

A comparison exercise concerning the fluid flow and heat transfer in a vertical channel asymmetrically heated at a constant heat flux has been initiated with the participation of eight teams coming from different laboratories. The dimensionless parameters were chosen so that a return flow occurs through the top section of the channel and four sets of conditions were proposed to model the open boundaries.

Both local and overall quantities were extracted from the numerical results: two expressions of the local Nusselt number along the heated plate, the local lengths of both the return flow and the associated recirculation, the local bulk temperature and four overall values defined by the average Nusselt numbers on the heated plate, the flow rate entering the channel through the bottom section and through the top section of the channel. All data provided by the participants were illustrated by histograms. The average, standard deviation and median statistical quantities were computed and reported on the figures. Comparisons show a relative good agreement between the different contributions.

For each of the four boundary conditions and the whole recorded variables, reference solutions have been defined. These numerical values have been gathered and copied out on Fig. 9(a)-(f), below their corresponding histograms. For benchmarking purpose, we recommend to compare at least the flow rates $q_{i n}(z=0)$ and $q_{i n}(z=A)$, and the different lengths of the recirculation $d_{w}(z)$ and $d_{\psi}(z)$ because these quantities are much sensitive to the boundary conditions than the thermal variables.

The effects of boundary conditions on fluid flow and heat transfer have been discussed. Whereas the local and mean Nusselt numbers based on the inlet temperature do not depend on the boundary conditions, the Nusselt number constructed on the bulk temperature reveals to be much sensitive to the conditions applied at the apertures. This large sensitivity is related to changes in flow patterns and flow rates. Just as a uniform zero pressure at the upper section of the channel promotes the return flow and its intensity, a constant pressure boundary condition to the lower aperture improve the inward flow rate. But whatever the air supply through the open sections, the fluid flow rates produced by the boundary layers along the heated surface are almost constant, what is in good agreement with the weak influence of the boundary conditions on heat transfer. Finally, we showed that the fluid flow is organized to minimize the total head loss. 
Even if the issue of finding the correct boundaries is out of the scope of this paper, the fully developed flow which appears in the lower adiabatic region when an uniform pressure is imposed in the lower open section is somewhat questionable. Therefore, local pressure (BC. 5b) at the lower aperture will be preferred to (BC. 5c) since it creates a non zero dynamic entry length.

\section{Appendix}

This exercise was originally organized in a french workshop for the Thermal French Society (SFT) in May 2004 by G. Desrayaud [24] and recently readdressed by the French Research Group AmeTH [33].

The main characteristics of the numerical schemes developed and/or used by the different teams are detailed below.

T1 The CFD code used by PPRIME is FDS [34]. The model solves numerically a form of the Navier-Stokes equations appropriate for low-speed, thermally-driven flow. The partial derivatives of the conservation equations of mass, momentum and energy are approximated as finite differences, and the solution is updated in time on a three-dimensional, rectilinear grid. The core algorithm is an explicit predictor-corrector scheme that is second order accurate in space and time. It approximates the governing equations on one or more rectilinear grids. The mesh used in this laminar $2 \mathrm{D}$ case is $400 \times 2560$ and the boundary conditions are exactly those describes in the bench excepted at the inlet/outlet where FDS used the "OPEN" boundary condition [34]. This condition assumes that the pressure perturbation $\widetilde{p}$ is zero at an outgoing boundary and $\widetilde{p}=-\rho_{\infty}|u|^{2} / 2$ at an incoming boundary. Notice that these boundary conditions are close to $G B-O$ boundary conditions described in this paper. The second difference with $G B-O$ is, as mentioned above, that the low Mach approximation is used instead of the Boussinesq approximation to solve the governing equations.

T2 Equations (1), (2) and (3) were solved either in their steady form for $L B$ and $L B-O$ problems, or in their transient form with a Crank-Nicolson scheme for cases $G B$ and $G B-0$. Spatial derivatives are expressed with a finite volume scheme with variables located at the center of the control volumes. Centered approximations are used for convective, advective and diffusive fluxes. A coupling between the mass equation (Eq. 1) and the pressure is enforced by applying a penalization technique on a coarser grid. The momentum nonlinear contribution and the pressure gradient are discretized so that the discrete kinetic energy balance mimics the behavior of its continuous counterpart; a similar condition is required for thermal equation. The discrete Navier-Stokes and energy equations, expressed in their stationnary form or at the new time step, are solved simultaneously in a large nonlinear system by the Newton-Raphson method. An under-relaxed procedure is used when time derivatives are dropped. Several meshes have been considered and the grid $253 \times 1520$ was finally retained. Additional details upon the numerical scheme and validation procedures can be found in [35-37].

T3 The Navier-Stokes equations under the Boussinesq assumptions are discretized by finite difference schemes. After time discretization, we obtain a system of Helmholtz equations. The 
velocity-pressure coupling is solved using a projection method which leads to a Poisson equation for the pressure correction. The variables $(u, w, p, T)$ are located on a staggered mesh and a spatial discretization scheme of second order is used. The Helmholtz and Poisson equations are solved respectively using TDMA and partial diagonalization. Moreover, the Poiseuille mode corresponding to the kernel of the Stokes operator is used to correct velocity and pressure to satisfy the boundary condition $G B-O$ at the inlet of the channel $[25,38]$. To implement $(G B, L B, L B-O)$, at the projection step, we impose Dirichlet boundary conditions on pressure at the inlet and outlet of the channel. Note that $G B-O$ is verified at each time step and $G B, L B, L B-O$ are verified only when the flow reaches the steady state [38].

T4 The usual dimensionless Boussinesq 2D Navier-Stokes equations were used. The time derivatives in the momentum and in the energy equations are performed by a second-order backward differentiation. The convection terms are approximate using a second-order AdamsBashford extrapolation method. The diffusion terms are implicitly treated. The resulting Helmholtz systems are solved by a direct solver. Pressure-velocity coupling is obtained by an incremental rotational projection method. A collocated finite volume method has been used. Details on numerical method can be found in [39]. The $G B-0$ case has been computed with a $48 \times 600$ grid size. The numerical code has been developed thanks to the environment OpenFOAM [40].

T5 The structure and solver of the computational code Aquilon@are issued from previous works [41], originally implemented with a Navier-Stokes finite volumes solver on the staggered MAC mesh and using the Uzawa augmented Lagrangian [42] method to deal with the divergence-free constraint. The discretization method with Discrete Operator Calculus is an extension of the MAC (Marker And Cell) method with staggered grids to unstructured meshes. The method is similar to Discrete Exterior Calculus based on differential geometry. The primal and dual meshes enable to express gradient, divergence, curl operators as well as Green, Gauss and Stokes theorems to obtain the continuum properties $\nabla \cdot(\nabla \wedge \vec{v})=0$ and $\nabla \wedge(\nabla f)=\overrightarrow{0}$ up to machine precision. The scheme is based on a node-center approach avoiding interpolations, where the scalar or vector components unknowns are distributed on nodes, faces and edges of the mesh stencils. The discretization is shown to locally and globally conserve up to machine precision, mass, kinetic energy and vorticity in the absence of viscosity. The spatial accuracy is found to be second-order on a structured or unstructured mesh including highly irregular meshes [43]. All the linear algebraic systems for the three methods, e.g. the prediction steps, are solved with the Krylov BiCGS tab2 algorithm preconditionned by the incomplete LU factorization of order zero ILU(0) [41-43]

T6 The code solves the unsteady two-dimensional Boussinesq equations in their elliptic form and has been developing at LIMSI. The temporal scheme adopted in order to discretize the Boussinesq equations is based on the second-order backward-Euler scheme for time advance and on the Adams-Bashforth extrapolation for convective terms. Thus, the diffusion terms are treated implicitly and the convective ones explicitly. The continuous problem is reduced to a discrete problem thanks to a finite volume method. The marker-and-cell (MAC) 
type staggered grid arrangement is used. All variables are spatially discretized with a second order centered scheme. The pressure-velocity coupled problem is solved by using a prediction-projection algorithm. The Poisson equation, derived from the continuity equation, is associated to homogeneous Neumann boundary condition at the whole boundaries (walls and artificial boundaries) [44]. Helmholtz systems are solved for the velocity fields using a GMRES algorithm and the Poisson equation is solved with a multi-grid algorithm. The governing equations have been integrated in time from fluid at rest up to reach the steady state. The size of the kernel for the unsteady Stokes operator, obtained by a singular value decomposition using the Lapack library, is equal to two. The two modes associated are the trivial (zero velocity, constant pressure) mode and a Poiseuille mode. The superposition principle $[25,30,31]$ has been used to obtain the numerical solution as a linear combination of a particular solution find with the finite volume scheme and both modes of the Stokes operator kernel. The result is obtained for a $256 \times 2048$ grid.

T7 The spatial discretization of the channel is achieved within a Finite Volume Method. The numerical code used is the commercial FLUENT code 12. Grid sensitivity has been studied an a non-uniform grid $(50 \times 300)$ was retained, with a refinement near the vertical walls as well as at the inlet and outlet boundaries. Numerical results were obtained from a pressurebased segregated solver, under Boussinesq approximation. Pressure-velocity coupling was solved using the SIMPLE algorithm. The Navier-Stokes equations were solved using, for the pressure discretization, the PRESTO (PRESsure STaggering Option) scheme, and for the momentum and energy equations, the QUICK scheme. The diffusion terms are centraldifferenced and are second order accurate.

Note that the dynamic boundary conditions are slightly different from $L B$ boundary conditions. Let us note $\vec{n}$ the unit normal vector pointing outward. Then, for fluid flows

- entering the channel, $\vec{v} \cdot \vec{n}<0, u=0$ and $(\partial w) /(\partial z)=-(\partial u) /(\partial x)$;

- leaving the channel, $\vec{v} \cdot \vec{n}>0,(\partial u) /(\partial z)=0$ and $(\partial w) /(\partial z)=-(\partial u) /(\partial x)$.

The other boundary conditions are the same as $L B$. It is worth to point out that $(\partial u) /(\partial x)$ is relatively small in the inlet/outlet sections, and then $(\partial w) /(\partial z) \approx 0$. Considering the no slip boundary conditions at walls and $(\partial u) /(\partial x) \approx 0$, we recover $u \approx 0$, namely the boundary conditions for $L B$.

T8 Both spatial and temporal discretization and the resolution procedure of discretized equations are identical to T6. The marker-and-cell (MAC) type staggered grid arrangement is used. The artificial boundaries of the computational domain which correspond to the inlet and the outlet of the channel are considered to be on the scalar pressure nodes. The pressure equation is associated to homogeneous Neumann boundary condition at the wall and Dirichlet boundaries conditions at the inlet and at the outlet so as to satisfy the local or the global Bernoulli condition for pressure. The four set of boundaries condition proposed in the benchmark have been treated. The whole results are obtained for a $128 \times 1300$ grid. 


\section{References}

[1] E. Bacharoudis, M. Vrachopoulos, M. Koukou, D. Margaris, A. Filios, S. Mavrommatis, Study of the natural convection phenomena inside a wall solar chimney with one wall adiabatic and one wall under a heat flux, Applied Thermal Eng. 27 (2007) 2266-2275.

[2] F. Incropera, Convection heat transfer in electronic equipment cooling, J. Heat Transfer 110 (1988) 1097-1111.

[3] R. Ben Yedder, E. Bilgen, Natural convection and conduction in trombe wall systems, Int. J. Heat Mass Transfer 34 (1991) 1237-1248.

[4] B. Balunov, A. Babykin, R. Rybin, B. Krylov, V. Tanchuck, S. Grigoriev, Heat transfer at mixed convection in vertical and inclined flat channels of the vacuum chamber of the iter international thermonuclear reactor, High Temperature 42 (2004) 126-133.

[5] W. Elenbaas, Heat dissipation of parallel plates by free convection, Physica 9 (1) (1942) 1-23.

[6] H. Sun, R. Li, E. Chénier, G. Lauriat, On the modeling of aiding mixed convection in vertical channels, Heat Mass Transfer (2012) 1-10.

[7] H. Sun, R. Li, E. Chénier, G. Lauriat, J. Padet, Optimal plate spacing for mixed convection from an array of vertical isothermal plates, International Journal of Thermal Sciences 55 (2012) 16 - 30.

[8] G. Desrayaud, A. Fichera, Laminar natural convection in a vertical isothermal channel with symmetric surfacemounted rectangular ribs, Int. J. Heat and Mass Transfer 23 (2002) 519-529.

[9] J. Hernandez, B. Zamora, Effects of variable properties and non-uniform heating on natural convection flows in vertical channels, Int. J. Heat and Mass Transfer 48 (2005) 793-807.

[10] Q. Lu, S. Qiu, G. Su, W. Tian, Z. Ye, Experimental research on heat transfer of natural convection in vertical rectangular channels with large aspect ratio, Experimental Thermal and Fluid Science 34 (1) (2010) 73 - 80.

[11] K. Ong, C. Chow, Performance of a solar chimney, Solar Energy 74 (1) (2003) 1 - 17.

[12] D. Ospir, C. Popa, C. Chereches, G. Polidori, S. Fohanno, Flow visualization of natural convection in a vertical channel with asymmetric heating, International Communications in Heat and Mass Transfer 39 (4) (2012) 486493.

[13] A. Andreozzi, B. Buonomo, O. Manca, Thermal management of a symmetrically of a heated channel-chimney system, International Journal of Thermal Sciences 48 (9) (2009) 742-748.

[14] J. Bodoia, J. Osterle, The development of free convection between heated vertical plates, ASME J. Heat Transfer 84 (1962) 40-44.

[15] T. Aihara, Effects of inlet boundary conditions on numerical solutions of free convection between vertical parallel plates, Report of the Institute of High Speed Mechanics, Tohuku University, Japan 28 (1973) 1-27.

[16] A. Dalbert, F. Penot, J. Peube, Convection naturelle laminaire dans un canal vertical chauffé à flux constant, Int. J. Heat Mass Transfer 24 (1981) 1463-1473.

[17] C. Kettleborough, Transient laminar free convection between heated vertical plates including entrance effets, Int. J. Heat Mass transfer 15 (1972) 883-896.

[18] D. Naylor, J. Floryan, J. Tarasuk, A numerical study of developing free convection between isothermal vertical plates, J. Heat Transfer 113 (1991) 620-626.

[19] H. Nakamura, Y. Asako, T. Naitou, Heat transfer by free convection between two parallel flat plates, Num Heat Transfer 5 (1982) 95-106.

[20] S. Boetcher, E. Sparrow, Buoyancy-induced flow in an open-ended cavity: Assessment of a similarity solution and of numerical simulation models, International Journal of Heat and Mass Transfer 52 (15-16) (2009) 3850 3856.

[21] A. Andreozzi, B. Buonomo, O. Manca, Thermal and fluid dynamic behaviors in symmetrical heated channelchimney systems, International Journal of Numerical Methods for Heat and Fluid Flow 20 (7) (2010) 811-833.

[22] S. Ramanathan, R. Kumar, Correlations for natural convection between heated vertical plates, J. Heat Transfer 113 (1991) 97-107.

[23] J. Liu, W. Tao, Numerical analysis of natural convection around a vertical channel in a rectangular enclosure, Heat and Mass Transfer 31 (1996) 313-321.

[24] G. Desrayaud, R. Bennacer, J.-P. Caltagirone, E. Chénier, A. Joulin, N. Laaroussi, K. Mojtabi, Etude numérique comparative des écoulements thermoconvectifs dans un canal vertical chauffé asymmétriquement, in: VIIIième Colloque Inter universitaire Franco-Québécois sur la Thermique des Systèmes, ART-06-14, 2007. 
[25] P. Le Quéré, On the computation of some external or partially enclosed natural convection flows (keynote paper), in: In 19th Int. Symp. Transport Phenomena, ISTP19, 2008, 8 pages, Reykjavik, Iceland.

[26] B. Webb, D. Hill, High Rayleigh number laminar natural convection in an asymmetrically heated vertical channel, J. Heat Transfer 111 (1989) 649-656.

[27] E. Sparrow, G. Chrysler, L. Azevedo, Observed flow reversals and measured-predicted nusselt numbers for natural convection in a one-sided heated vertical channel, ASME J. Heat Transfer 106 (1984) 325-334.

[28] R. Li, H. Sun, E. Chénier, G. Lauriat, Effet du rayonnement surfacique sur les recirculations de convection naturelle, in: Proc. of the annual Congrès Français de Thermique : Energie Solaire et Thermique, Vol. 19, Perpignan, 2011, pp. 181-186.

[29] R. Li, M. Bousetta, E. Chénier, G. Lauriat, Effect of surface radiation on natural convective flows and onset of flow reversal in asymmetrically heated vertical channels, International Journal of Thermal Sciences 65 (2013) 9-27.

[30] P. Le Quéré, On the computation of some external or partially enclosed natural convection flows, in: 7th International Conference on Computational Heat and Mass Transfer, 2011, paper 401, Istanbul, Turkey.

[31] C. Garnier, A. Sergent, P. Le Quéré, Modélisation numérique d'un canal vertical asymétriquement chauffé avec une condition aux limites à l'entrée de type vorticité, in: Proc. of the annual Congrès Français de Thermique, SFT Bordeaux, 2012.

[32] Y. Cherif, A. Joulin, L. Zalewski, S. Lassue, Outils pour l'étude expérimentale de la convection naturelle entre plaques planes verticales différentiellement chauffées, in: Proc. of the annual Congrès Français de Thermique, Vol. 18, 2010.

[33] E. Arquis, Rapport intermédiaire GDR AMETh: Simulation numérique de l'écoulement d'air dans un canal vertical partiellement chauffé. Exercice de comparaison (Mai 2010).

[34] Http://www.fire.nist.gov/fds.

[35] E. Chénier, R. Eymard, O. Touazi, Numerical results using a colocated finite volume scheme on unstructured grids for incompressible fluid flows, Numerical Heat Transfer Part B 49 (3) (2006) 259-276.

[36] O. Touazi, E. Chénier, R. Eymard, Simulation of natural convection with the collocated clustered finite volume scheme, Computers \& Fluids 37 (2008) 1138-1147.

[37] E. Chénier, R. Eymard, R. Herbin, A collocated finite volume scheme to solve free convection for general non-conforming grids, Journal of Computational Physics 228 (2009) 2296-2311.

[38] Z. Amine, S. Xin, S. Giroux-Julien, C. Ménézo, étude numérique des transferts thermo-convectifs dans un canal d'air vertical à flux imposé, in: Proc. of the annual Congrès Français de Thermique, SFT Perpignan, 2011, pp. $163-168$.

[39] R. Peyret, T. D. Taylor, Computational methods for fluid flow, Springer Series in Computational Physics, 1983.

[40] OpenFOAM 2.1, http ://www.openfoam.com, 2012.

[41] S. Vincent, J.-P. Caltagirone, P. Lubin, T. N. Randrianarivelo, An adaptative augmented lagrangian method for three-dimensional multimaterial flows, Computers \& Fluids 33 (2004) 1273-1289.

[42] M. Fortin, R. Glowinski, Méthodes de Lagrangien augmenté. Application à la résolution numérique de problèmes aux limites. Paris, Dunod, Dunod, 1982.

[43] P. Angot, J. P. Caltagirone, P. Fabrie, A fast vector penalty-projection method for incompressible nonhomogeneous or multiphase Navier-Stokes problems, Applied Mathematics Letters 25 (11) (2012) 1681-1688.

[44] E. Gadoin, P. Le Quére, O. Daube, A general methodology for investigating flow instabilities in complex geometries : application to natural convection in enclosure, International Journal for Numerical Methods in Fluids 37 (2001) 175-208. 


\section{List of Tables}

1 Notations for constructing the boundary conditions. . . . . . . . . . . . . 24

2 Some characteristics of the numerical codes and labels of teams. . . . . . . . . . 24 


\section{List of Figures}

1 Geometry and boundary conditions . . . . . . . . . . . . .

2 Fluid flow and temperature field for $L B$ boundary conditions (see Tab. 1). The vertical thick line located on the left wall emphasizes the heated section. (a) Streamlines and size of the recirculation at the top section, $d_{\psi}(z=A)(\mathrm{Eq} .14) ;(\mathrm{b})$ Horizontal component of the velocity, $u(x, z)$; (c) Vertical component of the velocity, $w(x, z)$, and size of the downward flow at the top section, $d_{w}(z=A)$ (Eq. 13);

(d) Temperature field. Data provided by team T2. . . . . . . . . . . . . . .

3 Histograms of results obtained for $G B-O$ (Tab. 1). Data provided by the different teams (Tab. 2) and statistical quantities: average, standard deviation and median values. . . . . . . . . . . . . . . . . . . . .

4 Histograms of results obtained for $L B-O$ (Tab. 1). Data provided by the different teams (Tab. 2) and statistical quantities: average, standard deviation and median values. . . . . . . . . . . . . . . . . . . .

5 Histograms of results obtained for $G B$ (Tab. 1). Data provided by the different teams (Tab. 2) and statistical quantities: average, standard deviation and median

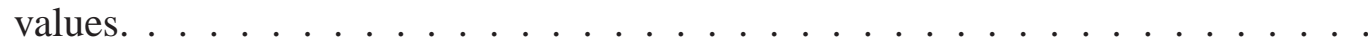

6 Histograms of results obtained for $L B$ (Tab. 1). Data provided by the different teams (Tab. 2) and statistical quantities: average, standard deviation and median values. . . . . . . . . . . . . . . . . . . .

7 Vertical component of the velocity at different horizontal sections of the channel for (a) $L B$ and (b) $G B$ boundary conditions (Tab. 1). The vertical thick line located on the left wall emphasizes the heated section. Data provided by team T2. . . . .

(a) Pressure and (b) vertical velocity profiles as a function of $z$ at mid-width of the channel for boundary conditions $L B, G B, L B-O$ and $G B-O$ (Tab. 1). Data provided by team T8. . . . . . . . . . . . . . . . . . . .

9 Histograms and error bars stand for average values $(\langle x\rangle)$ and standard deviations $\left(x_{\sigma}\right)$, for $L B-O, L B, G B-0$ and $G B$ (Tab. 1). Boxes are topped by the average values and the relative standard deviation $\left(x_{\sigma} /\langle x\rangle\right)$ reported in parentheses and expressed in permillage. Values below the graphics are the reference solutions which are set to the median values. . . . . . . . . . . . . . . . . . . 


\begin{tabular}{|l|c|c|c|}
\hline \multicolumn{2}{|c|}{} & \multicolumn{2}{c|}{ Channel Inlet } \\
\cline { 3 - 4 } \multicolumn{2}{|c|}{} & Relation 5b & Relation 5c \\
\hline Channel & Relation 6c & $L B$ & $G B$ \\
\cline { 2 - 4 } Outlet & Relation 6d & $L B-0$ & $G B-0$ \\
\hline
\end{tabular}

Table 1: Notations for constructing the boundary conditions.

\begin{tabular}{|c|c|c|c|c|c|}
\hline Team & Laboratory & Code & Grid & Stationary scheme & Boundary conditions \\
\hline T1 & PPRIME & FDS [34] & $400 \times 2560$ & no & $G B-0$ \\
\hline $\mathrm{T} 2$ & MSME, LAMA & Laboratory code [35-37] & $253 \times 1520$ & yes/no & $G B, G B-O, L B, L B-O$ \\
\hline $\mathrm{T} 3$ & CETHIL & Laboratory code $[25,38]$ & $200 \times 1200$ & no & $G B, G B-O, L B, L B-O$ \\
\hline $\mathrm{T} 4$ & PIMENT, LaSIE & Laboratory code [39] (with OpenFoam [40]) & $48 \times 600$ & no & $G B-O$ \\
\hline T5 & I2M-TREFLE & Aquilon $\mathbb{R}[41-43]$ & $100 \times 1000$ & no & $G B-O$ \\
\hline T6 & LIMSI & Laboratory code $[25,30,31]$ & $256 \times 2048$ & no & $G B-O$ \\
\hline $\mathrm{T} 7$ & LGCgE, LEME & Fluent $(\mathbb{R}$ & $50 \times 300$ & yes & $L B$ \\
\hline $\mathrm{T} 8$ & LIMSI & Laboratory code & $128 \times 1300$ & no & $G B, G B-O, L B, L B-O$ \\
\hline
\end{tabular}

Table 2: Some characteristics of the numerical codes and labels of teams. 


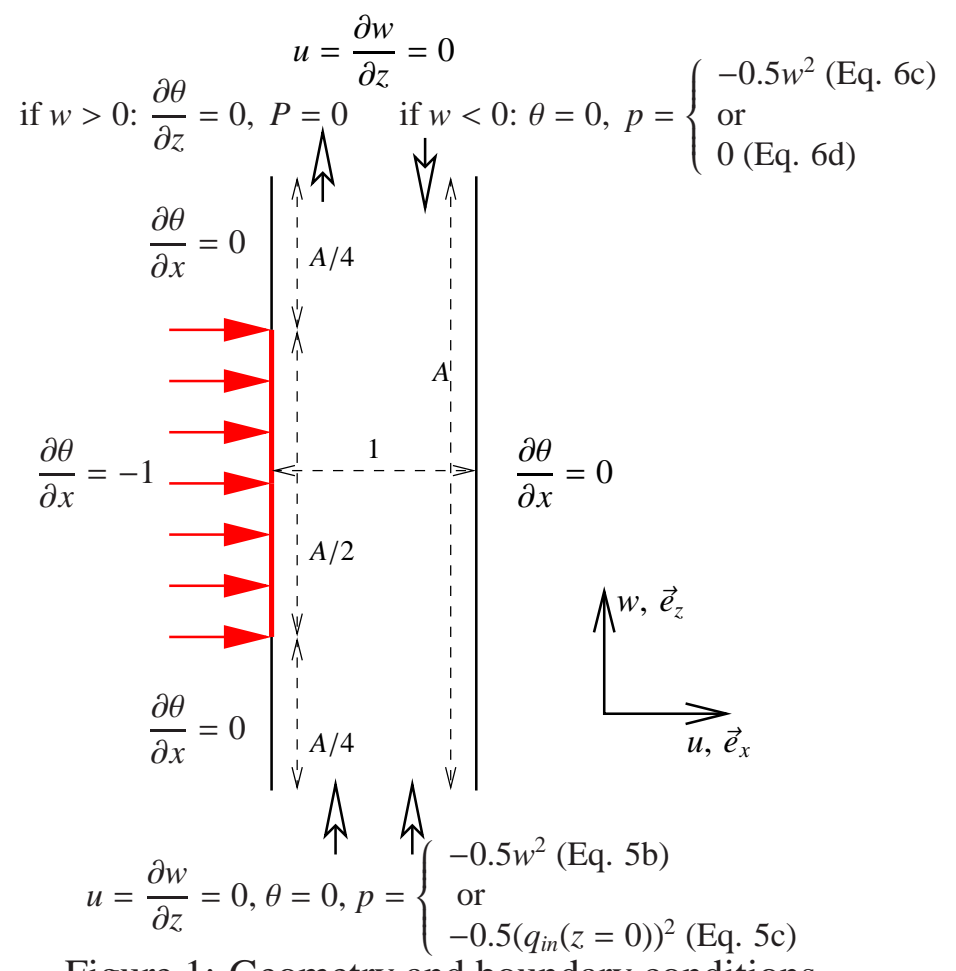

Figure 1: Geometry and boundary conditions 


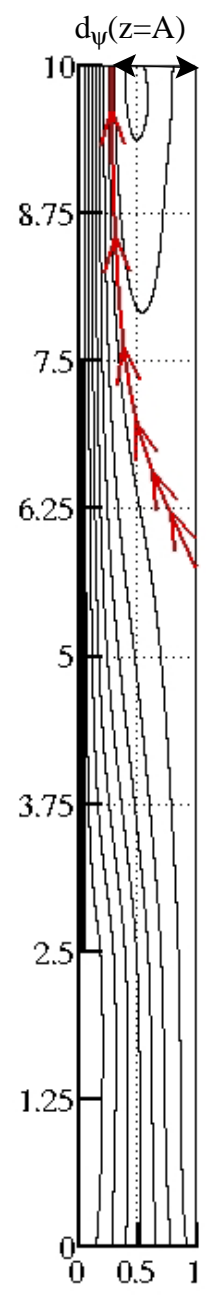

(a)

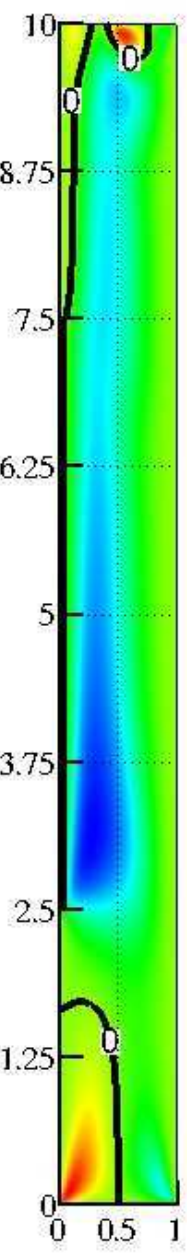

(b)

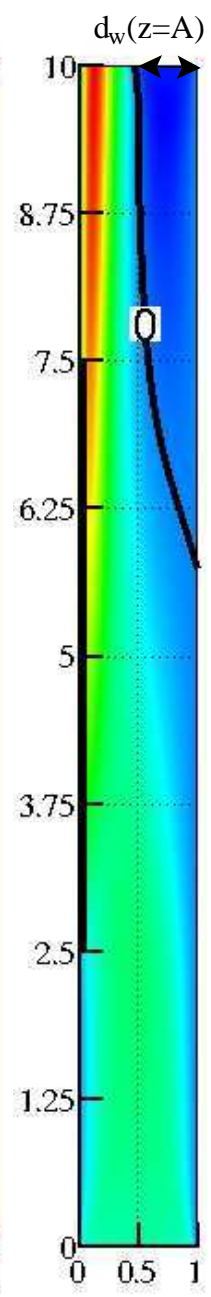

(c)

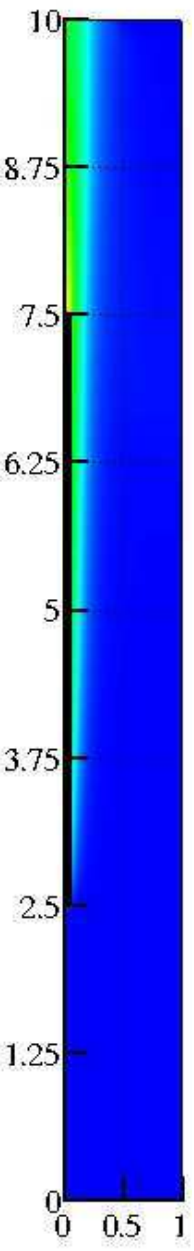

(d)

Figure 2: Fluid flow and temperature field for $L B$ boundary conditions (see Tab. 1). The vertical thick line located on the left wall emphasizes the heated section. (a) Streamlines and size of the recirculation at the top section, $d_{\psi}(z=A)$ (Eq. 14); (b) Horizontal component of the velocity, $u(x, z)$; (c) Vertical component of the velocity, $w(x, z)$, and size of the downward flow at the top section, $d_{w}(z=A)$ (Eq. 13); (d) Temperature field. Data provided by team T2. 

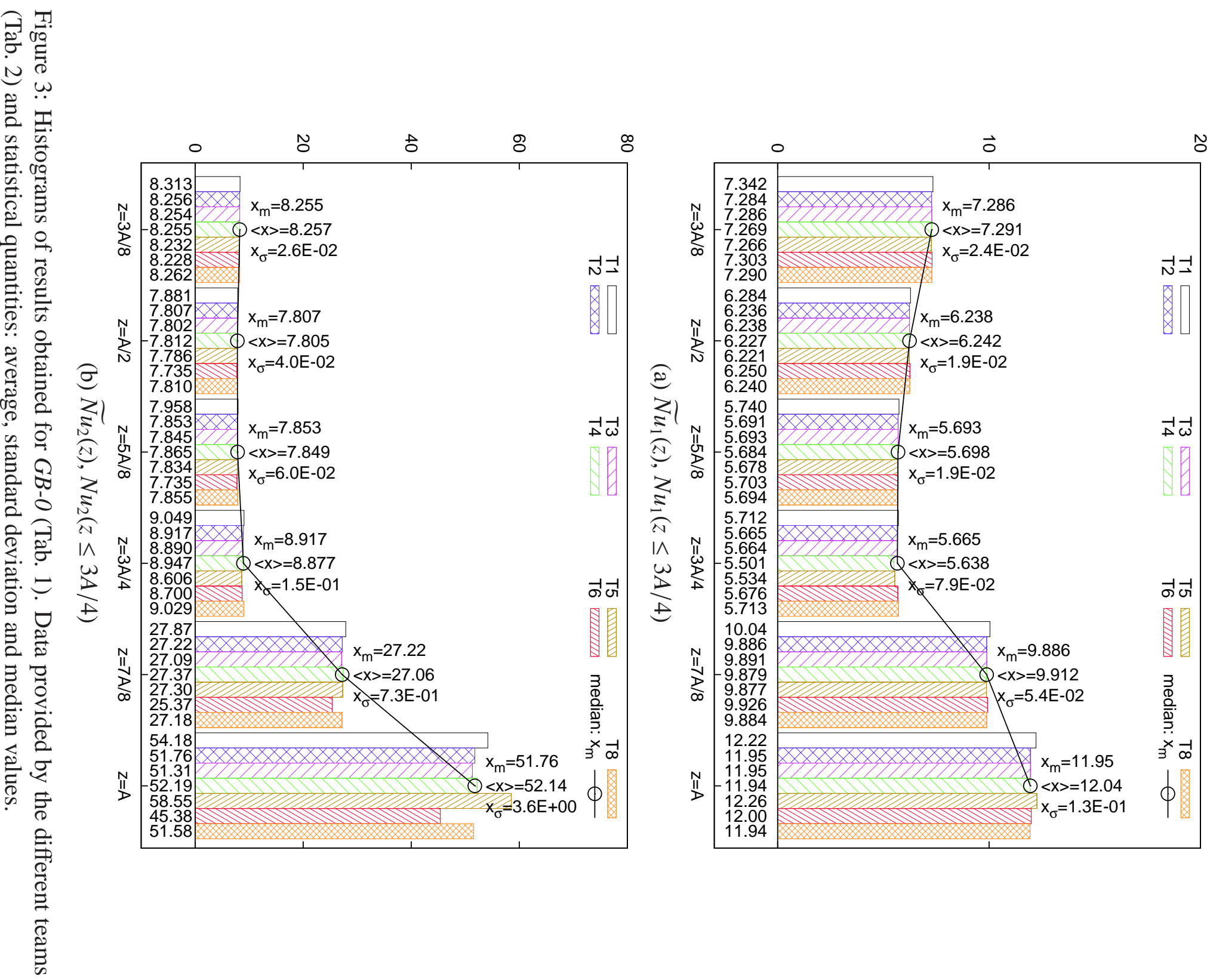


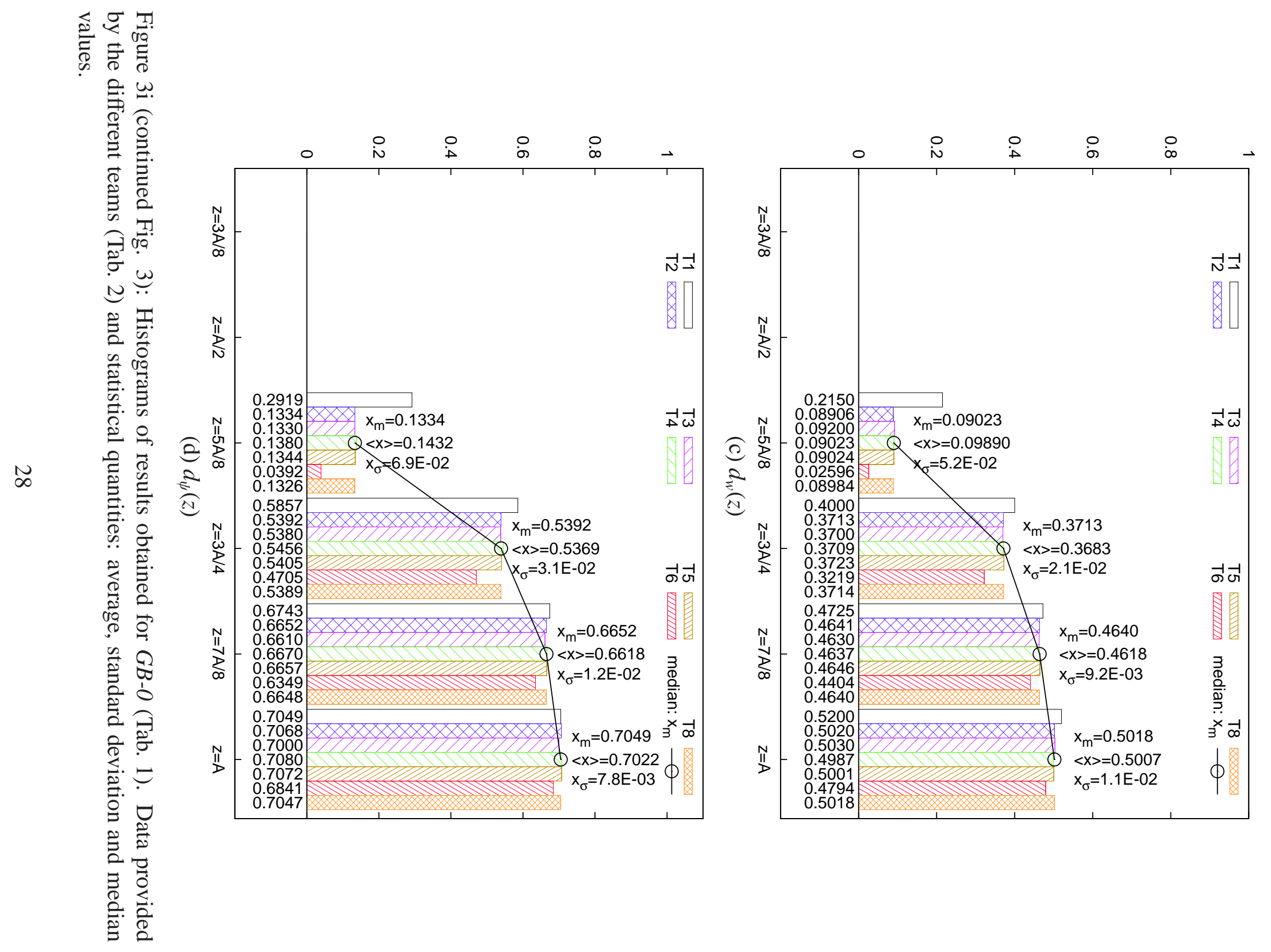




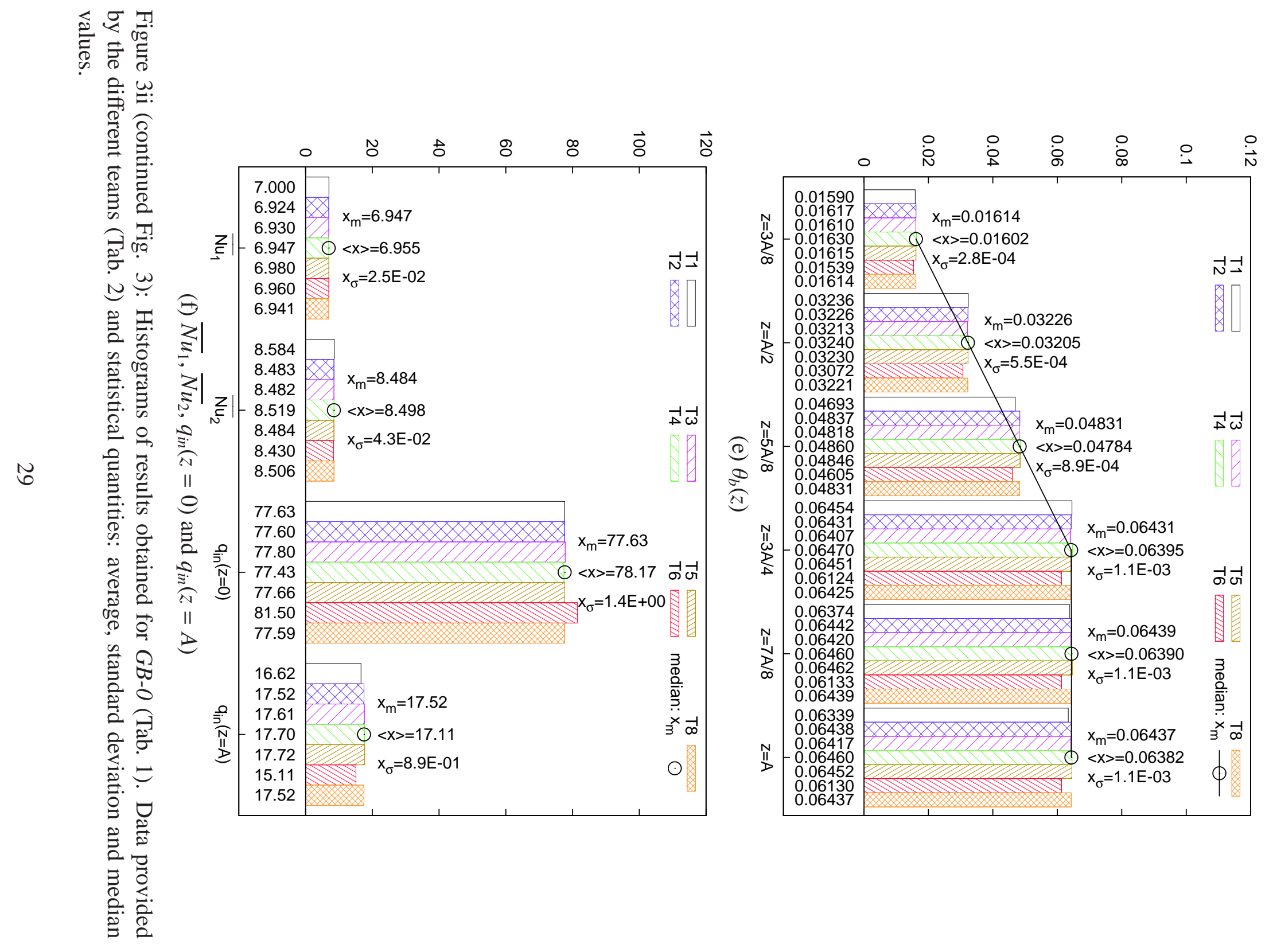



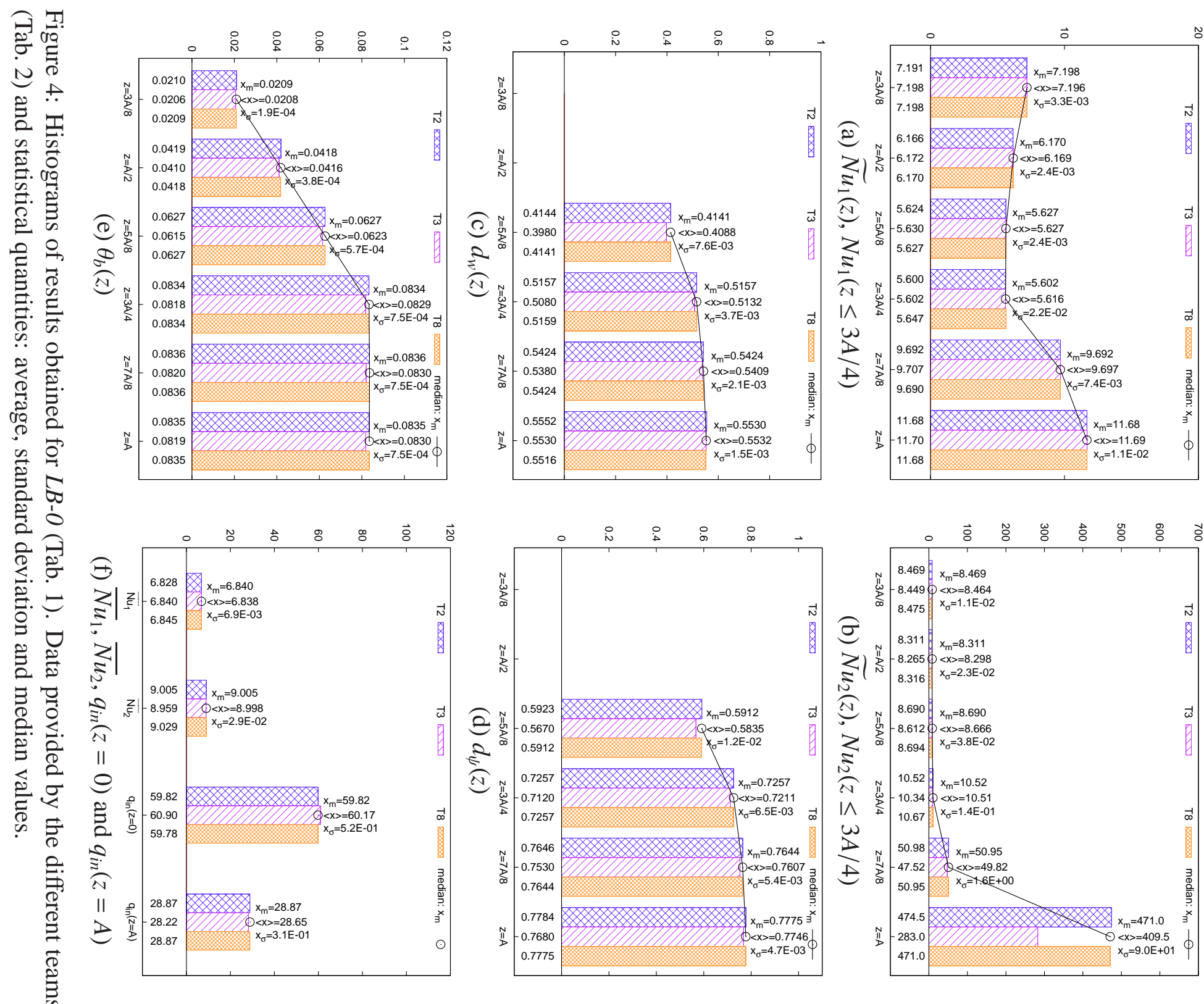

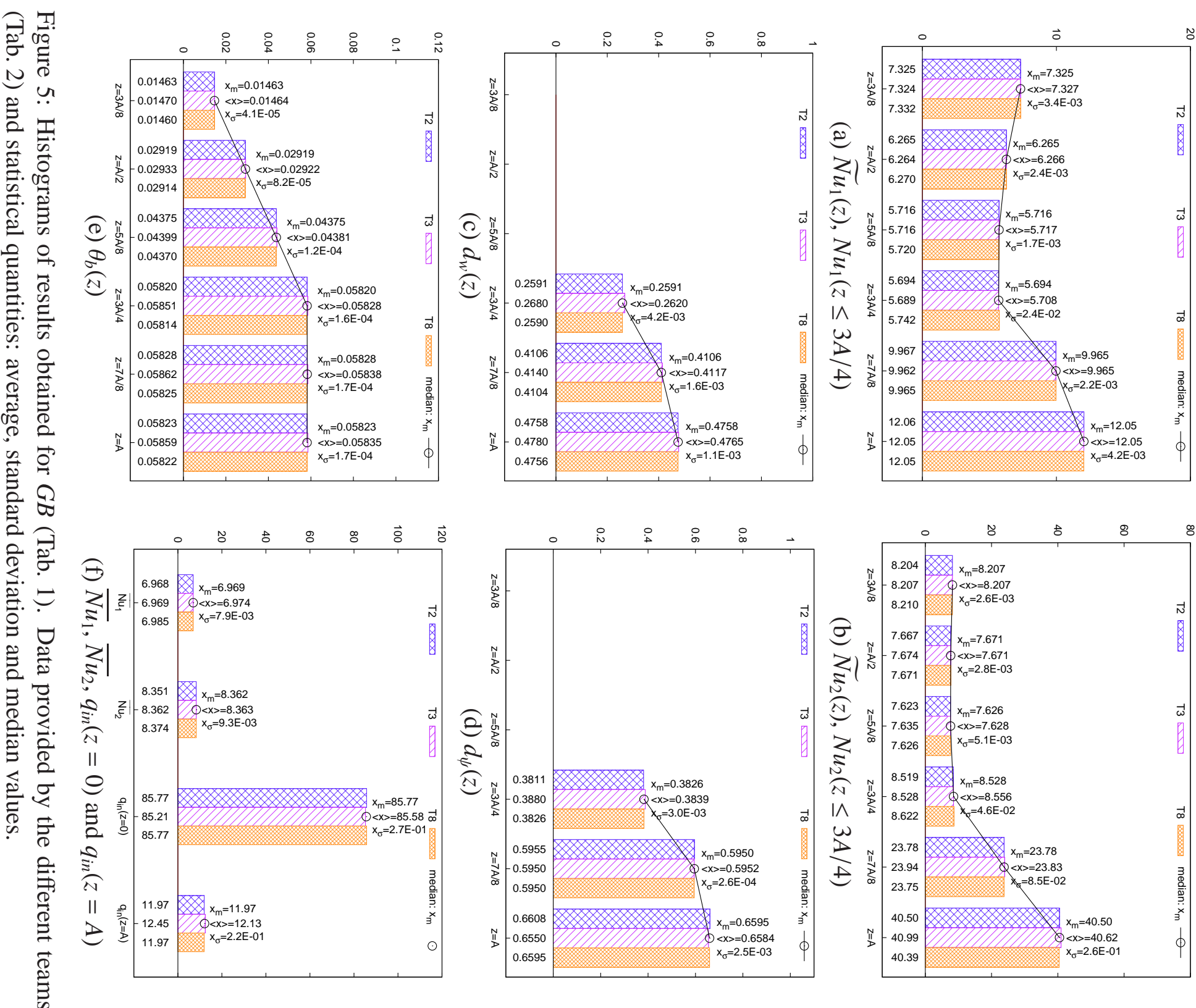

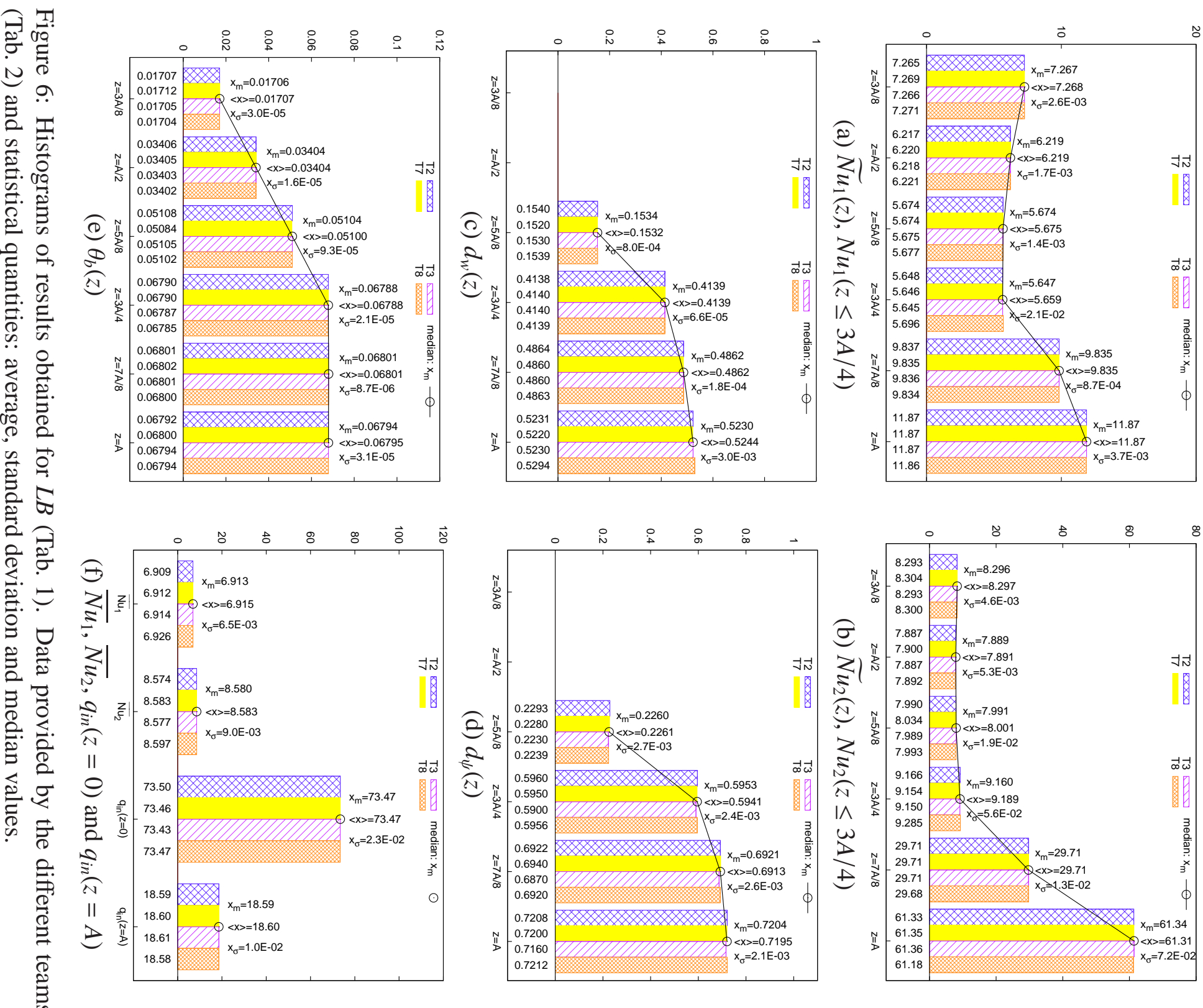


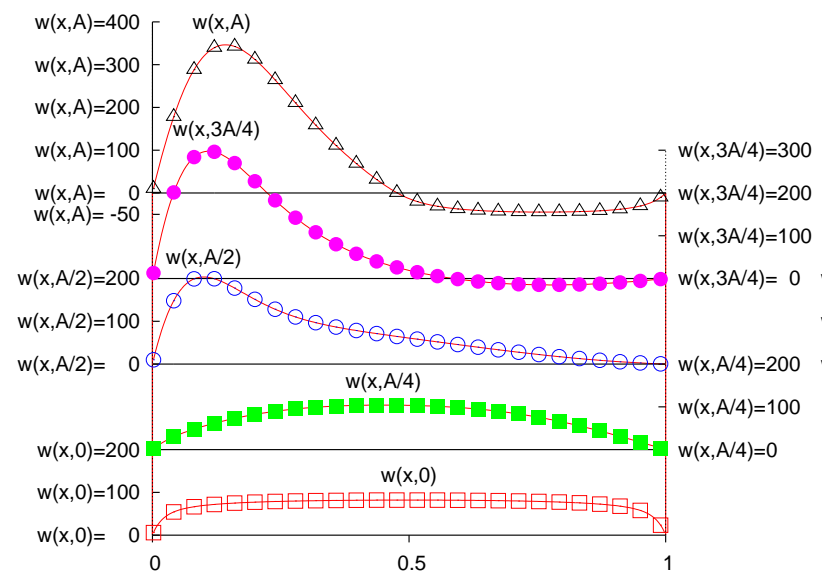

(a) $L B$

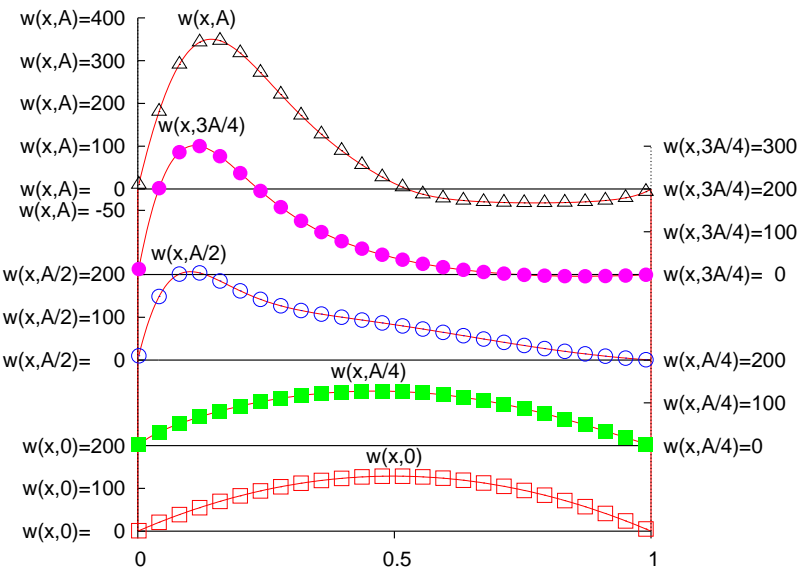

(b) $G B$

Figure 7: Vertical component of the velocity at different horizontal sections of the channel for (a) $L B$ and (b) $G B$ boundary conditions (Tab. 1). The vertical thick line located on the left wall emphasizes the heated section. Data provided by team T2.

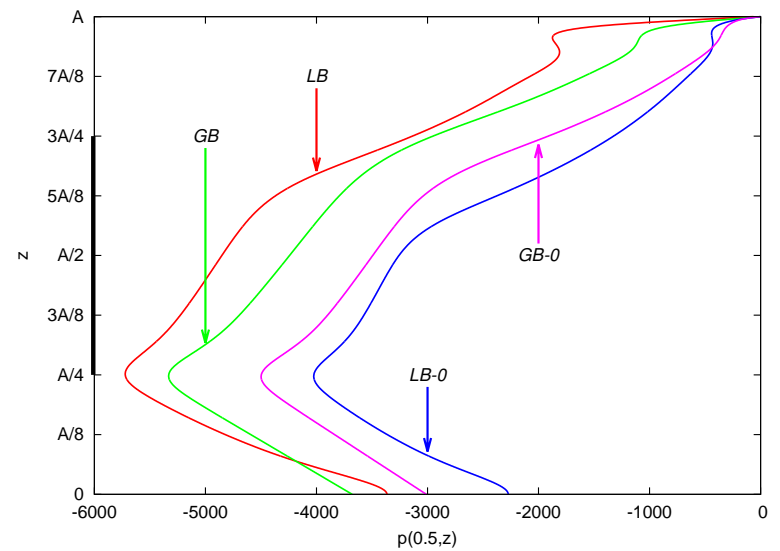

(a) Pressure

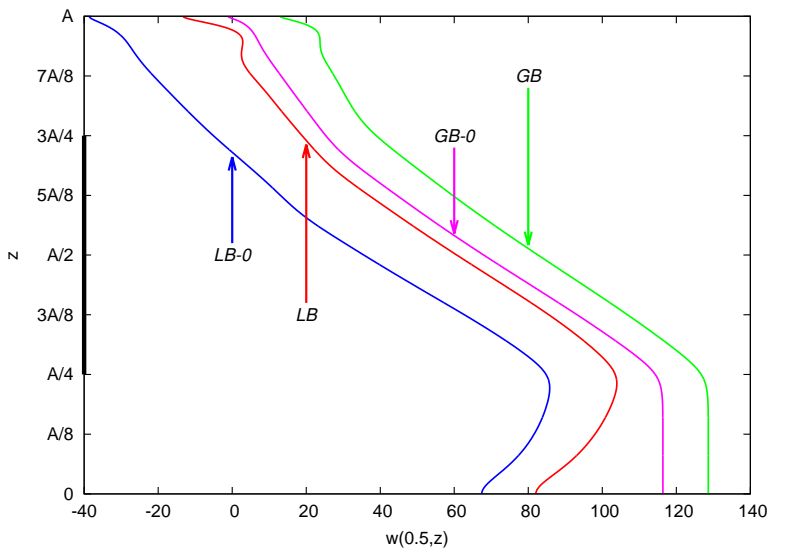

(b) Vertical component of the velocity

Figure 8: (a) Pressure and (b) vertical velocity profiles as a function of $z$ at mid-width of the channel for boundary conditions $L B, G B, L B-O$ and $G B-O$ (Tab. 1). Data provided by team T8. 

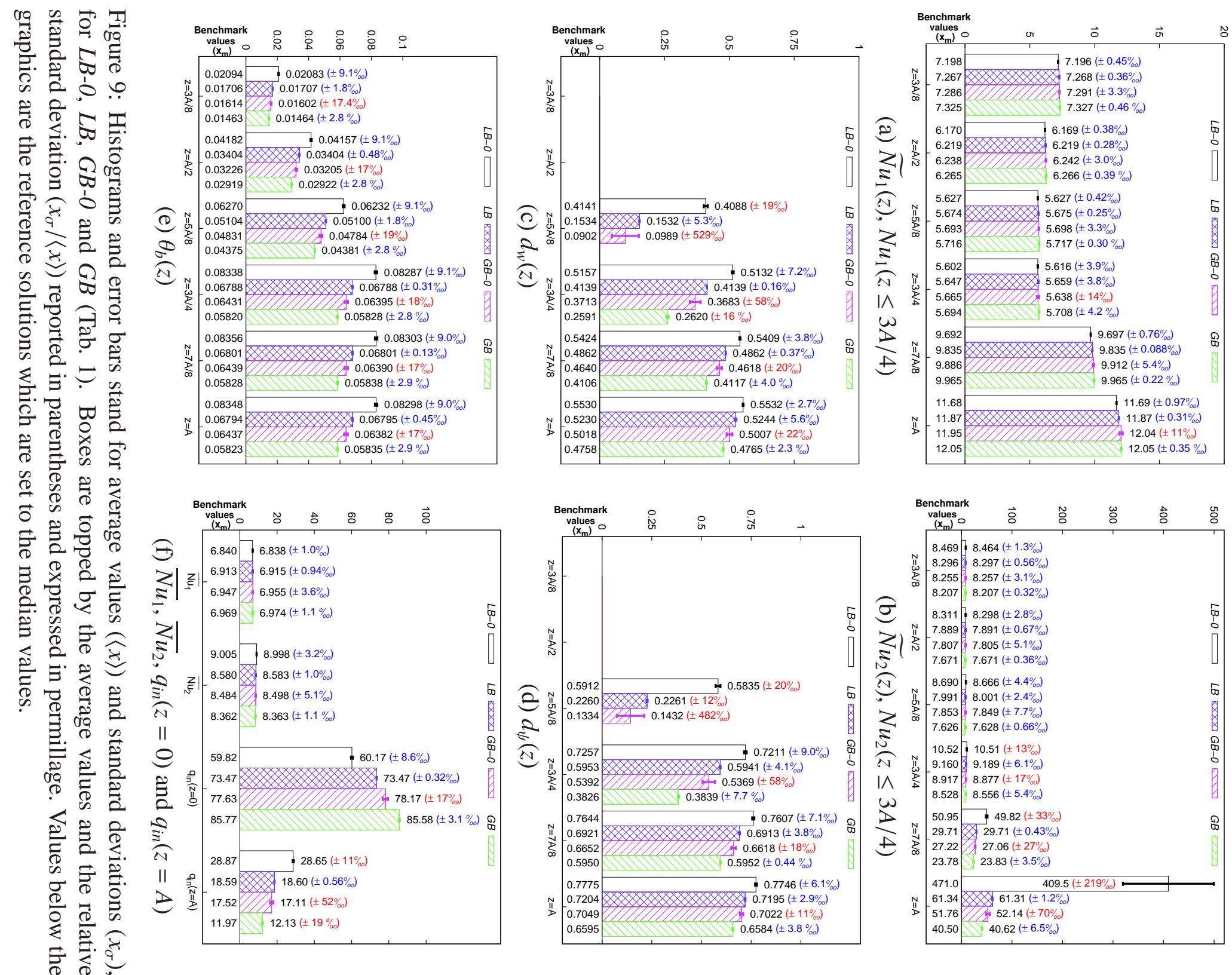